\title{
Norois
}

Environnement, aménagement, société

\section{Colmatage sédimentaire des marais maritimes et variations relatives du niveau marin au cours des 6000 dernières années en rade de Brest (Finistère)}

Sedimentary infilling of salt-marshes and relative sea-level variations during the last 6000 years in the Bay of Brest (western Brittany)

\section{Pierre Stéphan}

\section{OpenEdition \\ Journals}

\section{Édition électronique}

URL : https://journals.openedition.org/norois/3659

DOI : $10.4000 /$ norois.3659

ISSN : $1760-8546$

\section{Éditeur}

Presses universitaires de Rennes

\section{Édition imprimée}

Date de publication : 30 novembre 2011

Pagination : 9-37

ISBN : 978-2-7535-1765-3

ISSN : 0029-182X

\section{Référence électronique}

Pierre Stéphan, «Colmatage sédimentaire des marais maritimes et variations relatives du niveau marin au cours des 6000 dernières années en rade de Brest (Finistère) », Norois [En ligne], 220 | 2011, mis en ligne le 30 novembre 2013, consulté le 13 janvier 2022. URL : http://journals.openedition.org/ norois/3659; DOI : https://doi.org/10.4000/norois.3659

Ce document a été généré automatiquement le 13 janvier 2022.

(c) Tous droits réservés 


\title{
Colmatage sédimentaire des marais maritimes et variations relatives $d u$ niveau marin au cours des 6000 dernières années en rade de Brest (Finistère)
}

\author{
Sedimentary infilling of salt-marshes and relative sea-level variations during \\ the last 6000 years in the Bay of Brest (western Brittany)
}

Pierre Stéphan

1 De nombreuses études des variations relatives du niveau marin à l'Holocène ont été réalisées sur les côtes françaises de l'Atlantique et de la Manche (Fairbridge, 1961; Morzadec-Kerfourn, 1969, 1974, 1975, 1985, 1995, 2005 ; Delibrias et Guillier, 1971 ; Delibrias et al., 1971 ; Mariette, 1971 ; Ters, 1973, 1986 ; Pirazzoli, 1976, 1996 ; Jones et al., 1987 ; Van de Plassche, 1991 ; Guilcher, 1992 ; Regnauld et al., 1996 ; Lambeck, 1997) et de la Méditerranée (L'Homer et al., 1981 ; Laborel et al., 1994 ; Morhange et al., 1996; Lambeck et Bard, 2000 ; Vella et Provansal, 2000 ; Morhange et al., 2001 ; Vella, 2004 ; Vella et al., 2005). Ces travaux se basent sur un ensemble de datations radiocarbone effectuées à partir d'un matériel varié : tourbe littorale ou dulçaquicole, charbons de bois, vase marine, bio-constructions, coquilles, objets et vestiges archéologiques.

2 Avant $10000 \mathrm{BP}$, aucun de ces témoins ne permet d'estimer la position du niveau marin sur les côtes de la Manche, de l'Atlantique et de la Méditerranée. Toutefois, les observations disponibles sur le plateau continental témoignent d'un bas niveau marin, situé à 100-120 m sous le niveau actuel, entre 20000 et 18000 BP (Pinot, 1968, 1973; Hinschberger, 1970 ; Delanoë et Pinot, 1977). Vers 15000 BP s'amorce la transgression post-glaciaire à un rythme important jusqu'à $6000 \mathrm{BP}$. Dès lors, le niveau marin atteint une position proche de l'actuelle et le mouvement transgressif connaît un ralentissement important (fig. 1 - planche I). Une tendance similaire est également 
observée sur les côtes voisines de l'Angleterre (Waller et al., 1999 ; Shennan et Horton, 2002 ; Boomer et Horton, 2006) et de la Belgique (Denys et Baeteman, 1995). À partir de $6000 \mathrm{BP}$, il semble que les vitesses de remontée du niveau marin soient assez variables selon les régions littorales considérées.

Sur la façade méditerranéenne française, les faibles marnages permettent des reconstitutions très précises des variations du niveau marin relatif depuis $6000 \mathrm{BP}$, par le biais d'indicateurs biologiques (Laborel et al., 1994 ; Morhange et al., 1996, 2001 ; Vella et Provansal, 2000). Les balanes offrent ainsi une précision de $\pm 20 \mathrm{~cm}$, tandis que les tourbes dulçaquicoles du delta du Rhône et les corniches à Lithophyllum lichenoides permettent de positionner le niveau marin relatif avec une précision moyenne de \pm $35 \mathrm{~cm}$. Les courbes obtenues montrent ainsi une élévation régulière du niveau marin relatif entre 6300 et $4000 \mathrm{BP}$, à une vitesse d'environ $2 \mathrm{~mm} / \mathrm{an}$ (fig. 1 - planche I). À partir de 4000 et $3500 \mathrm{BP}$, un stationnement autour de $-2 \mathrm{~m}$ durant plus d'un millénaire est observé par C. Vella et M. Provansal (2000), avant d'être remis en question par C. Morhange et al. (2001). De 3000 à $1500 \mathrm{BP}$, l'élévation se poursuit sans oscillation importante à un rythme plus lent, estimé à $0,7 \mathrm{~mm} / \mathrm{an}$. Enfin, le dernier millénaire de notre ère est caractérisé par une certaine stabilité du niveau relatif de la mer (fig. 1 - planche I).

Figure 1 : Positions du niveau marin et tendances déterminées à partir de différents témoins sédimentaires, biologiques et archéologiques sur les façades méditerranéennes et MancheAtlantique-Mer du Nord françaises, belges et anglaises/Sea-level index points and trend along the French, Belgian and south-England coasts
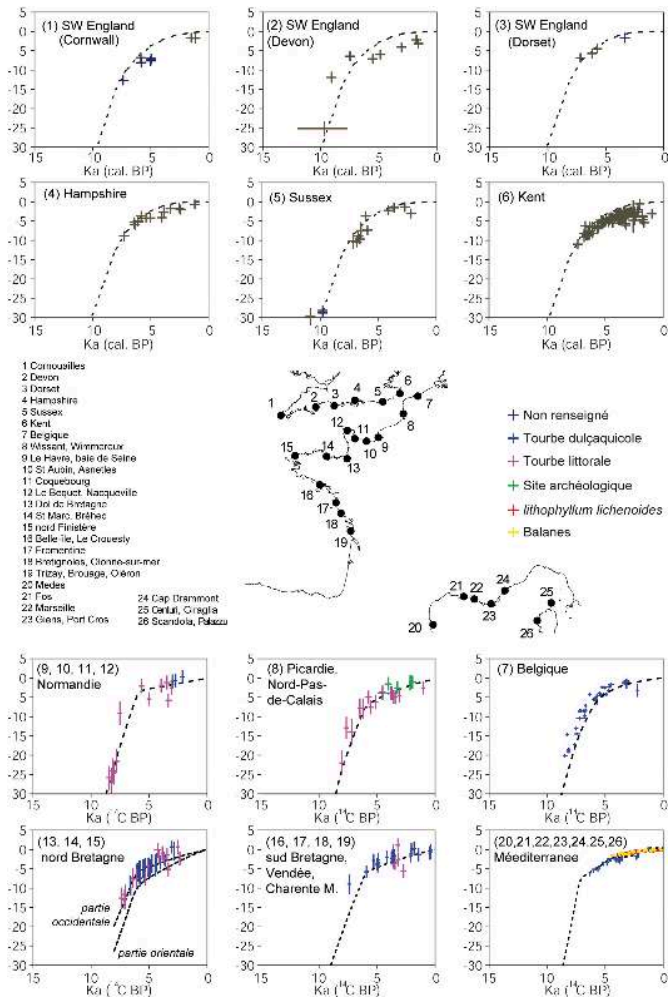

(d'après Shennan et Horton, 2002 ; Denys et Baeteman, 1995 ; Lambeck, 1997 ; Aloisi et al., 1978 ; Laborel et al., 1994 ; Morhange et al., 1996, 2001 ; Vella et Provansal, 2000)

4 Sur les rivages de la Manche et de l'Atlantique, l'amplitude de la marée introduit une marge d'incertitude bien plus importante dans le positionnement des anciens niveaux marins. Les reconstitutions se basent principalement sur les niveaux de tourbe littorale 
dont la position d'origine par rapport au niveau marin est fixée arbitrairement entre les pleines mers moyennes (PMM) et les plus hautes mers de vive-eau (PMVE) (Lambeck, 1997). Dans ce cas, les anciens niveaux marins ne peuvent être positionnés qu'avec une précision minimale de $\pm 1 \mathrm{~m}$ dans la plupart des secteurs côtiers de la Manche et de l'Atlantique.

5 À cette incertitude s'ajoute également celle liée au tassement des niveaux tourbeux après leur dépôt qui peut conduire à sous-estimer la véritable position du niveau marin (Denys et Baeteman, 1995; Vella, 1999 ; Vella et Provansal, 2000 ; Long et al., 2006 ; Edwards, 2006). Toutefois, diverses méthodologies ont été développées au cours des dix dernières années pour améliorer la précision des reconstitutions. La plupart d'entre elles s'appuient sur des bio-indicateurs tels que les foraminifères, les diatomées ou les amibes et privilégient une approche quantitative en utilisant des outils statistiques tels que les fonctions de transfert (Zong et Horton, 1999 ; Gehrels, 1999 ; Horton et al., 2000 ; Edwards et Horton, 2000, 2006 ; Gehrels et al., 2001 ; Edwards et al., 2004 ; Southall et al., 2006 ; Boomer et Horton, 2006 ; Massey et al., 2006 ; Woodroffe, 2009).

Les variations relatives du niveau marin au cours des 6000 dernières années restent toutefois difficiles à établir de manière aussi précise qu'en Méditerranée. En outre, les travaux de K. Lambeck (1997) ont mis en évidence des écarts importants dans le rythme de la transgression marine le long des côtes de Vendée, de Bretagne, de Normandie et de Picardie. Les courbes dressées pour ces différentes régions côtières se superposent difficilement en raison de phénomènes glacio-hydro-isostatiques. En fonction de la configuration de leur plate-forme continentale et de leur éloignement plus ou moins important par rapport aux grands inlandsis nord-européens, les différentes régions littorales françaises ont été affectées par des phénomènes crustaux d'ampleur variable. Les nombreuses études menées sur les côtes anglaises insistent fortement sur ce point (Waller et Long, 2003 ; Massey et al., 2008). La synthèse, réalisée par I. Shennan et B. Horton (2002), des dates radiocarbone acquises au cours des trois dernières décennies sur l'ensemble des côtes anglaises, met en avant les écarts importants observés dans les variations holocènes du niveau marin relatif. Tandis que la partie nord de l'Angleterre, soumise à un soulèvement lié au réajustement glacio-isostatique, a connu une baisse du niveau marin relatif au cours des 6000 dernières années, la partie sud a montré une tendance inverse sous l'effet conjugué d'une faible subsidence et d'une remontée globale du niveau de la mer. Aussi, est-il préférable de privilégier une approche régionale, voire locale, des variations holocènes du niveau marin relatif.

En Bretagne occidentale, les données disponibles se limitent aux travaux de M.Th. Morzadec-Kerfourn portant sur l'étude des tourbes d'estran affleurant sur les plages du nord Finistère (Morzadec-Kerfourn, 1969, 1974). Par conséquent, toutes les interprétations géomorphologiques concernant les anciennes positions de la ligne de rivage découlent de ces travaux qui n'ont, à ce jour, jamais été réactualisés. L'objectif de la présente étude est donc d'apporter des données nouvelles sur les anciennes positions du niveau marin et de proposer une courbe des variations relatives du niveau de la mer à l'Holocène pour la partie occidentale de la Bretagne, en s'appuyant sur l'étude du colmatage sédimentaire de trois marais maritimes d'arrière-cordon situés en rade de Brest. La méthodologie utilisée s'appuie sur une quinzaine de datations radiocarbone et le recours aux analyses litho- et bio-stratigraphiques des dépôts sédimentaires côtiers. Une fonction de transfert basée sur les foraminifères a été employée pour déterminer les anciennes positions du niveau marin. 


\section{Les sites d'étude}

8 La Bretagne apparaît comme une région particulièrement propice à l'étude des variations relatives du niveau marin. L'ensemble du Massif armoricain est, en effet, considéré comme tectoniquement stable depuis le début de l'Holocène (Ters, 1973; Morzadec-Kerfourn, 1995). Par conséquent, les variations relatives du niveau de la mer sont en majeure partie d'origine eustatique dans cette région, certainement combinées à une composante glacio-hydro-isostatique. Il faut toutefois souligner que la partie occidentale de la péninsule armoricaine semble affectée par un lent soulèvement depuis quelques millénaires, estimé à $0,01 \mathrm{~mm} / \mathrm{an}$ (Lenotre, 1994, in Regnauld et al., 1996). En outre, P. Pirazzoli (1976) rappelle qu'au cours du Quaternaire, les variations de charge liées aux fluctuations eustatiques ont pu provoquer de fortes contraintes dans l'écorce terrestre près des côtes, même dans les régions connues pour n'être pas le siège d'une activité tectonique récente. Des tremblements de terre isolés ont pu en résulter, accompagnés parfois de quelques déplacements verticaux sur certaines portions littorales. La littérature fait état de plusieurs séismes survenus durant la période historique (Grellet et al., 1993). Toutefois, ces événements se concentrent essentiellement le long des grandes failles hercyniennes, parfois réactivées, dont le tracé reste assez éloigné de nos secteurs d'étude situés en rade de Brest (Van VlietLanoë et al., 1997).

9 La rade de Brest se présente sous la forme d'un vaste plan d'eau qui communique avec la mer d'Iroise par un étroit goulet, large de seulement $1,7 \mathrm{~km}$. Cette configuration limite fortement l'entrée des houles océaniques et confère à la rade un caractère d'abri particulièrement prononcé (fig. 2). Les vagues les plus morphogènes sont donc générées par le vent local et leur hauteur ne dépasse jamais un mètre en raison des faibles longueurs de fetch. Le marnage maximum théorique est de $7,6 \mathrm{~m}$. L'hydrodynamisme réduit qui caractérise ce secteur a néanmoins permis l'édification d'une trentaine de cordons littoraux, isolant de la mer de nombreux marais maritimes de faibles dimensions (Guilcher et al., 1957 ; Stéphan, 2008).

Trois d'entre eux, situés dans la partie orientale de la rade, ont été étudiés en détail (fig. 3A, 3B, 3C). Ces marais présentent de grandes similitudes d'un point de vue morphosédimentaire. Ils couvrent des superficies relativement faibles, comprises entre 3 ha pour les marais de Porzguen et d'Arun et 6 ha pour le marais de Troaon. Ces marais se situent en aval de bassins-versants peu étendus, drainés par des cours d'eau intermittents. Ils sont situés à une altitude comprise entre 3,5 et $4 \mathrm{~m} \mathrm{NGF}$, correspondant au niveau des pleines mers de vive-eau moyennes et sont parcourus par un réseau de chenaux de marée particulièrement encaissés dans le schorre. Leur surface est marquée par la présence de salt pans, dénuées de végétation, caractéristiques des hauts schorres. D'une manière générale, deux ceintures végétales peuvent être distinguées. Une ceinture à Halimione portulacoides, Puccinellia Maritima, Plantago maritima couvre la plus grande partie de ces marais, tandis que la partie la plus interne est occupée par une roselière à phragmites et une jonçaie à Scirpus maritimus. En surface, le matériel sédimentaire est principalement constitué d'une vase grise argilo-silteuse (grain moyen compris entre 20 et $40 \mu \mathrm{m}$ ). Un enrichissement en éléments grossiers (grain moyen voisin de $300 \mu \mathrm{m}$ ) est observé dans le lit des chenaux de marée principaux, empruntés quotidiennement par les courants de flot et le jusant. 
Les cordons littoraux qui protègent ces trois marais se composent d'un matériel sablograveleux (grain moyen compris entre 10 et $20 \mathrm{~mm}$ ). Ces flèches présentent une seule crête végétalisée dont l'altitude dépasse de quelques décimètres seulement le niveau atteint par les plus hautes mers. Par conséquent, elles sont susceptibles de connaître occasionnellement des phénomènes de débordement par les vagues de tempête.

11 Le marais de Lanveur (fig. 3D) présente des caractéristiques morphosédimentaires quelque peu différentes. Bien qu'il bénéficie de conditions d'abri particulièrement prononcées, il n'est protégé par aucun cordon littoral. Le schorre occupe le fond de la baie et forme une ceinture végétale d'une centaine de mètres de large, colonisée par Puccinellia maritima et par l'Obione dans sa partie haute, et par Spartina alterniflora dans sa partie basse. Une large slikke forme le bas d'estran, parcourue par un réseau hiérarchisé de chenaux de marée.

\section{Méthodologie}

\section{Carottages et levés de terrain}

Dans le cadre de cette étude, 31 sondages ont été réalisés à la tarière à gouge, dans les marais maritimes de Porzguen, de Troaon et d'Arun (fig. 3) afin de reconstituer la stratigraphie des dépôts sédimentaires. La position des sondages a été relevée au GPS différentiel et l'altitude a été raccordée au niveau NGF à partir de bornes géodésiques situées à proximité des secteurs d'étude. Les analyses sédimentologiques et les datations radiocarbone ont été réalisées à partir d'échantillons prélevés tous les $10 \mathrm{~cm}$ le long de quatre sondages de "référence ». Au total, 155 échantillons ont été récoltés sur le terrain et analysés en laboratoire. 
Figure 2: Localisation des secteurs d'étude/Location of studied site

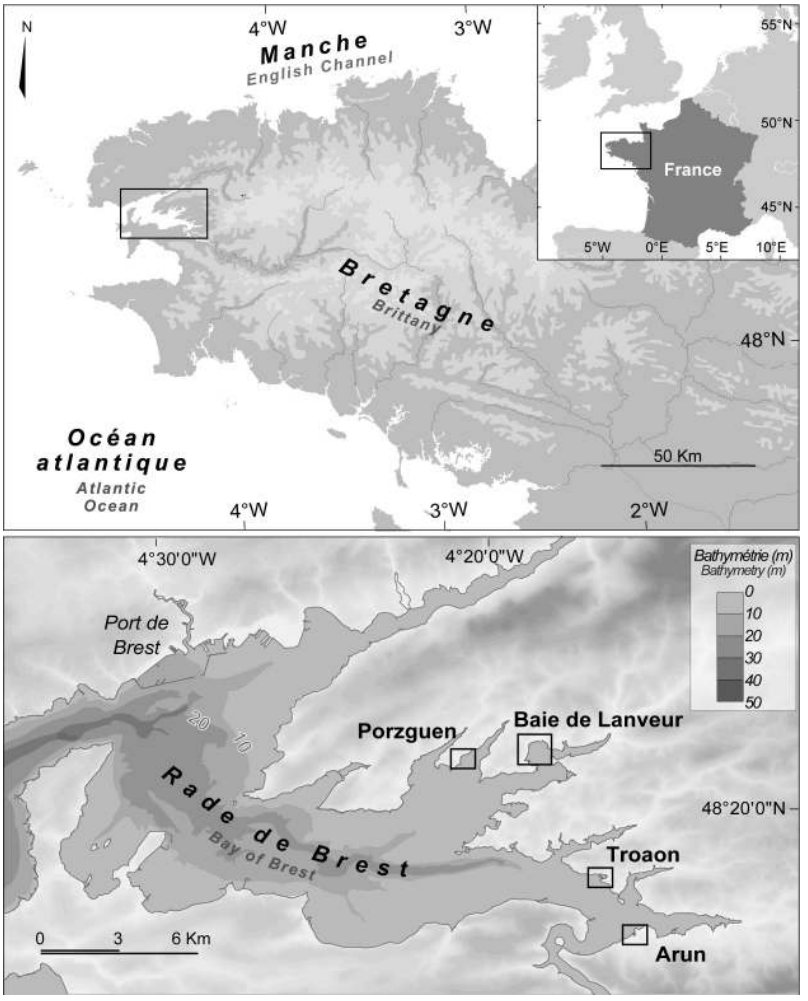

Figure 3 : Localisation des secteurs d'étude, des points de carottage et des transects utilisés pour étudier la répartition altitudinale des foraminifères/Location of boreholes and transects in Bay of Brest

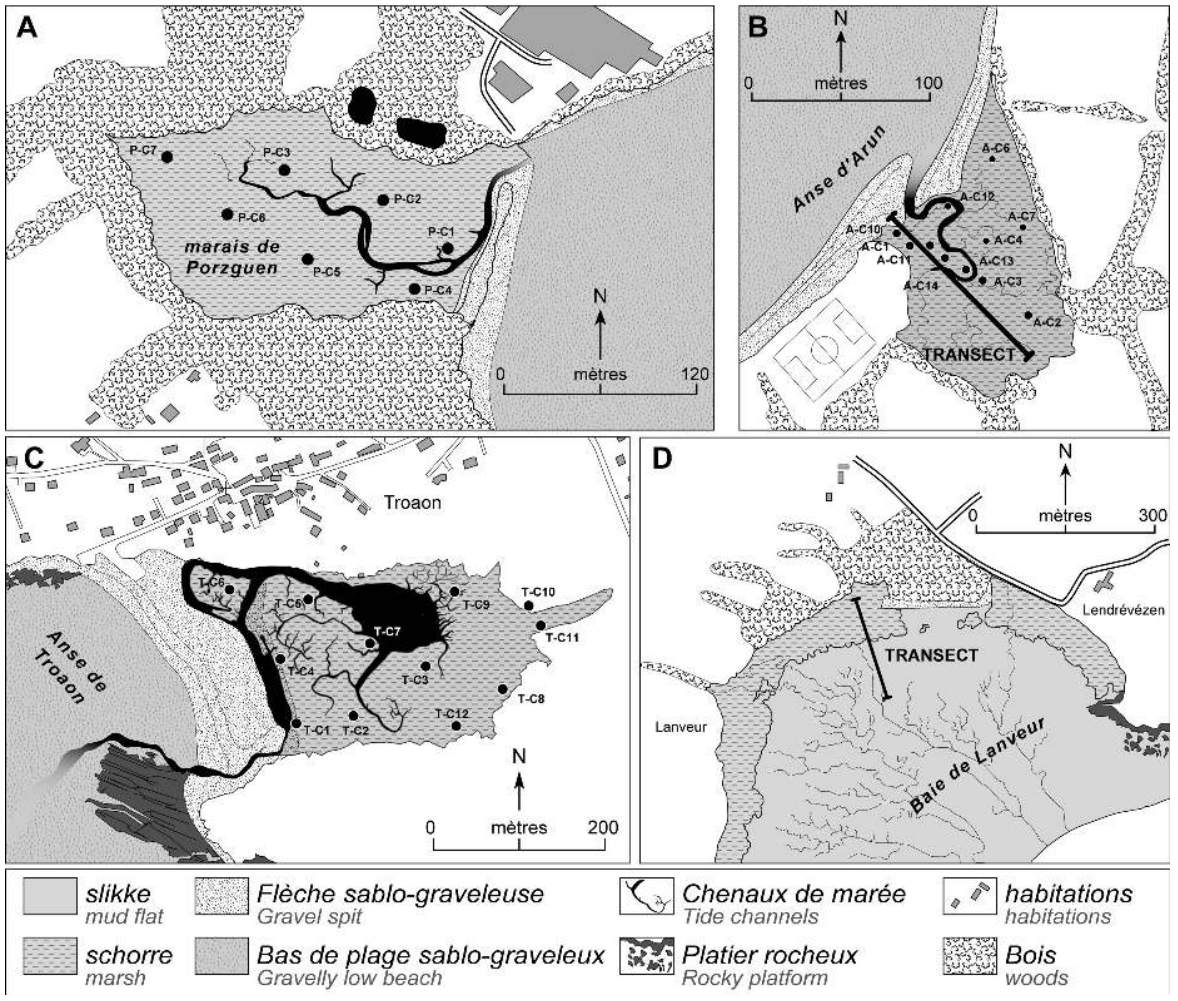




\section{Analyse sédimentologique et microfaunistique}

13 L'ensemble des échantillons prélevés sur le terrain a fait l'objet d'une analyse granulométrique et microfaunistique. L'analyse granulométrique des vases a été réalisée au laboratoire de sédimentologie de l'Ifremer à l'aide d'un granulomètre laser, tandis que les sables et les graviers ont fait l'objet d'un tamisage.

14 L'analyse microfaunistique a porté uniquement sur les foraminifères. Après tamisage humide, seule la fraction comprise entre $63 \mu \mathrm{m}$ et $500 \mu \mathrm{m}$ a été retenue, comme le proposent J. W. Murray (1991) et B. P. Horton et R. J. Edwards (2006). Pour chaque échantillon, environ 300 individus de foraminifères ont été comptés à la loupe binoculaire. Cette quantité est considérée comme statistiquement suffisante pour l'étude des espèces les plus abondantes (Fatela et Taborda, 2002). Les espèces ont été identifiées suivant la classification générique de R. Loeblich et $\mathrm{H}$. Tappan (1988) et de J. W. Murray (1971). Dans le cas des Elphidium, les différents écophénotypes ont été regroupés sous un seul nom d'espèce appelé Elphidium spp. L'abondance relative de chaque espèce a été exprimée en pourcentage de la population totale (abondance fractionnelle).

\section{Développement et application d'une fonction de transfert basée sur les foraminifères}

15 Fréquemment utilisés comme marqueurs stratigraphiques holocènes, les foraminifères fournissent des indications précises sur les paléoenvironnements (Dubar et Anthony, 1995 ; Long et Hughes, 1995 ; Long et al., 1999; Lespez et al., 2002). Leur très grande abondance et leur excellent potentiel de conservation en font un outil efficace pour les reconstitutions basées sur l'écologie des espèces actuelles, au même titre que d'autres groupes taxonomiques, tels que les mollusques. Les études menées sur la répartition des foraminifères benthiques dans les environnements de haute slikke et de schorre (Scott et Medioli, 1978, 1980 ; Scott et al., 1996; Horton et al., 2000) ont montré qu'il existe un étagement vertical des principales espèces permettant de les utiliser comme indicateurs du niveau marin.

16 Les fonctions de transfert utilisant les foraminifères ont été utilisées pour la première fois par J. Guilbault et al. (1996) dans le cadre de recherches sur le niveau marin au Canada. Depuis, de nombreuses études menées dans les marais maritimes de la zone tempérée ont testé et affiné cette approche dans l'objectif de reconstituer les variations du niveau marin relatif, avec une haute résolution pour la période holocène (Gehrels, 1999 ; Horton et al., 2000 ; Edwards et Horton, 2000, 2006 ; Edwards et al., 2004 ; Southall et al., 2006; Boomer et Horton, 2006 ; Massey et al., 2006). Bien que les techniques statistiques utilisées varient d'une étude à une autre, la méthode générale reste grossièrement la même et se subdivise en trois étapes.

17 La première étape comprend l'échantillonnage, l'analyse des foraminifères actuels et l'examen de leur répartition altitudinale. L'objectif est alors de produire un modèle statistique de distribution altitudinale des foraminifères en relation avec les niveaux de marée. Dans cette étude, la distribution des foraminifères présents à la surface du sédiment a été étudiée à partir de deux transects situés dans le marais maritime d'Arun (fig. 3C) et en baie de Lanveur (fig. 3D), censés fournir un modèle de distribution valable pour l'ensemble de la rade. Ces deux marais ont été choisis car ils présentent des 
morphologies différentes et permettent ainsi d'appréhender une large gamme de conditions environnementales.

Au total, 53 échantillons (fig. 4) d'un volume d'environ $10 \mathrm{~cm}^{3}$ de sédiments ont été prélevés sur le terrain à l'aide d'une tarière manuelle sur les dix premiers centimètres de profondeur, comme le recommandent R. T. Patterson et al. (1999), G. Duchemin et al. (2005) et A. C. Massey et al. (2006). L'altitude des points d'échantillonnage a été relevée au DGPS et raccordée au système NGF (IGN 69) à partir de points géoréférencés situés à proximité de nos sites. Les altitudes ont ensuite été converties en valeur standard, tenant compte des conditions marégraphiques du site. Pour ce faire, nous avons utilisé "l'indice du niveau marin standardisé », ou SWLI (Standardised Water Level Index), défini de la façon suivante (Edwards et Horton, 2000) :

SWLI $=([($ Alt. Echantillon $-\mathrm{NM}) /(\mathrm{PM} 95-\mathrm{NM})] \times 100)+100$

où NM est le niveau moyen de la mer (soit 0,38 m NGF en rade de Brest) et PM95 est le niveau des pleines mers de vive-eau moyenne de coefficient 95 (soit 3,31 m NGF en rade de Brest). Les foraminifères présents au sein de ces échantillons ont fait l'objet d'un comptage en laboratoire en suivant la même méthode que celle décrite précédemment. Notons qu'aucune distinction n'a été faite entre les individus morts (thanatocénose) et les individus vivants (biocénose). Contrairement aux travaux de R. J. Edwards et al. (2004), E. Armynot du Châtelet et al. (2005), K. E. Southall et al. (2006), B. P. Horton et R. J. Edwards (2006), la prise en compte des assemblages totaux a été préférée car elle présente l'avantage d'intégrer les cycles saisonniers et pluri-annuels affectant les peuplements de foraminifères et représente plus précisément les conditions environnementales générales (Scott et Medioli, 1980).

Figure 4: Répartition des foraminifères le long des transects réalisés dans les marais de Lanveur et Arun en rade de Brest/Foraminiferal abundances in Lanveur and Arun salt marshes. Transect elevation, sediment samples and vegetation zones are also outlined

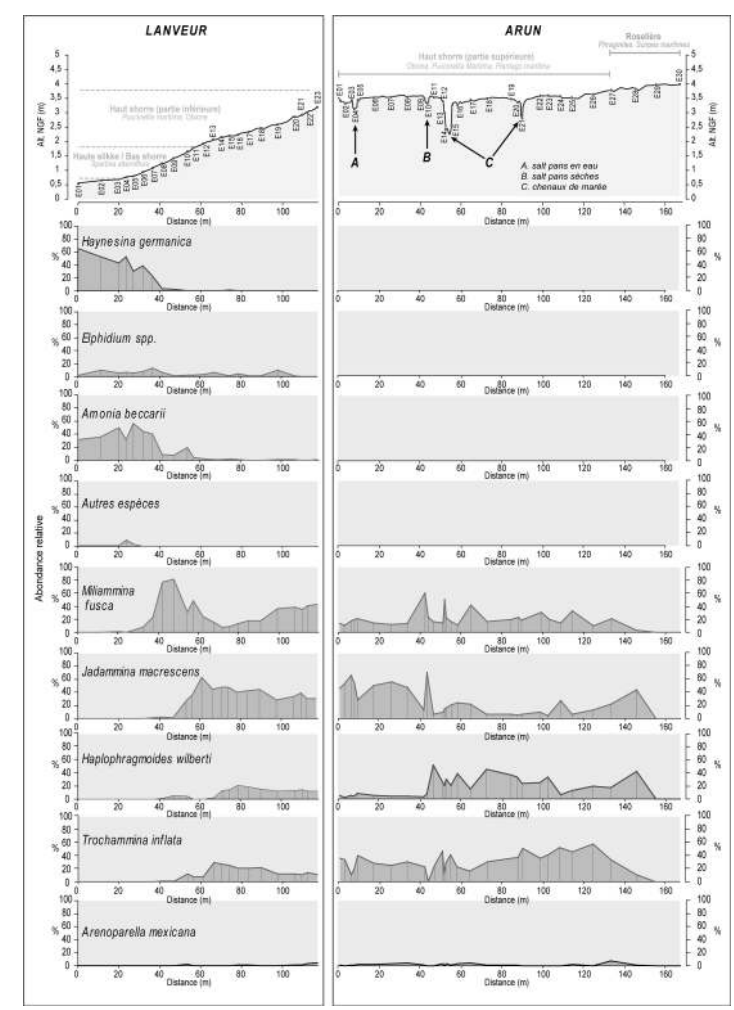


21 Dans une seconde étape, ces données ont été utilisées pour produire une série de relations statistiques entre les divers taxons de foraminifères et le niveau de marée. Les analyses statistiques ont été réalisées sous le logiciel C² (version 1.3) (Juggings, 2003). La méthode de régression WA-PLS (Weighted-averaging partial least squares) a été choisie car elle offre les plus forts coefficients de détermination $\left(\mathrm{r}^{2}=0,79\right)$ et de très faibles écartstypes de prédiction (Root Mean Square Error of Prediction, ou RMSEP $=0,51 \mathrm{~m}$ ). En définitive, la fonction de transfert ainsi obtenue permet de prédire la position du niveau marin à $\pm 51 \mathrm{~cm}$.

Dans une troisième étape, la fonction de transfert a été appliquée aux assemblages de foraminifères fossiles reconnus dans les 155 échantillons prélevés le long des sondages de référence. Sur la base de l'abondance relative des espèces présentes, le modèle statistique a permis d'attribuer une position relative du niveau marin à chaque échantillon. La fiabilité des estimations a également été déterminée de manière statistique en utilisant la technique MAT (Modern Analogue Technique), via le programme $\mathrm{C}^{2}$. Cette technique identifie les échantillons fossiles qui n'ont pas d'équivalent dans les échantillons utilisés pour mettre au point la fonction de transfert et permet, le cas échéant, de ne pas en tenir compte dans l'interprétation des résultats. La technique est basée sur le calcul d'un coefficient de similitude, obtenu par la comparaison de chaque échantillon fossile avec les dix échantillons les plus proches utilisés dans la fonction de transfert. Le $10^{\mathrm{e}}$ percentile de ce coefficient constitue un seuil approximatif pour estimer si les échantillons fossiles disposent d'équivalents actuels (Horton et al., 2000 ; Edward et Horton, 2000 ; Horton et Edwards, 2005 ; Southall et al., 2006).

\section{Datations radiocarbone}

Dans cette étude, dix-sept datations radiocarbone AMS ont été réalisées au laboratoire AMS-Labor du Physikalisches Institut d'Erlangen (Friedrich Alexander Universität) en Allemagne (tableau 1). Elles ont été obtenues sur des échantillons prélevés à différents niveaux des quatre sondages de référence. Les âges mesurés sur les sédiments correspondent aux âges conventionnels, exprimés en années BP («Before Present» c'est-à-dire avant 1950). Ils ont été corrigés par calibration au moyen du logiciel Calib 6.0 (Stuiver et Reimer, 1993) grâce à la courbe IntCal09 de P.J. Reimer et al. (2009). Les dates calibrées sont complétées par un écart-type 掼 qui signifie qu'elles ont 95,4\% de chance de se situer dans l'intervalle \pm 2 [5

\section{Résultats}

\section{Le marais de Troaon : description de la stratigraphie et des unités stratigraphiques}

Le marais de Troaon montre une séquence transgressive type, se traduisant par une accentuation du caractère marin des dépôts mis en place successivement vers la terre ferme, sous l'effet de la transgression fini-holocène (fig. 5A). Trois unités stratigraphiques ont pu être distinguées.

L'unité 1 repose sur le toit du substratum constitué de schistes dévoniens (fig. 5). Elle est composée de plusieurs niveaux tourbeux, riches en morceaux de bois et en débris de phragmites. En faible proportion, la fraction minérale est argilo-silteuse et présente un 
grain moyen compris entre 40 et $70 \mu \mathrm{m}$. L'épaisseur de ces dépôts organiques atteint $1,7 \mathrm{~m}$ au niveau du sondage T-C2 (fig. 5A). Les datations réalisées à la base et au sommet de cette unité s'échelonnent entre 6250 cal.BP et 5070 cal.BP, soit de la fin de l'Atlantique au début du Subboréal. Ces dépôts sont donc contemporains des tourbes qui affleurent au niveau des plus basses mers de vive-eau en Bretagne (MorzadecKerfourn, 1969 ; Visset et Bernard, 2006) et dont la mise en place est associée à un contexte de ralentissement de la transgression marine. L'analyse des foraminifères indique un niveau de sédimentation situé autour de 3,5 $\pm 0,52 \mathrm{~m}$ au-dessus du niveau moyen de la mer (fig. 6), correspondant à la limite entre l'étage supralittoral et le milieu terrestre, généralement occupée par les phragmites et Scirpus maritimus. Les assemblages sont dominés par Trochammina inflata et, secondairement, par Jadammina macrescens et Haplophragmoides wilberti. Deux niveaux tourbeux (unité 1B et 1D, fig. 6), dans lesquels les foraminifères sont totalement absents, témoignent d'un environnement de dépôt soustrait à l'influence marine et correspondent à une tourbe dulçaquicole. Le passage à un milieu plus continentalisé peut être attribué à un léger mouvement régressif, ou encore à l'exhaussement progressif de la tourbière littorale par sédimentation organique. L'unité 1 se termine par un niveau de vase tourbeuse (unité 1F) qui témoigne d'une influence marine accrue, probablement liée à une reprise de la transgression marine.

Tableau 1 : Datations radiocarbone et positionnement du niveau marin en rade de Brest/ Radiocarbon dates and sea-level index points obtained in Bay of Brest

\begin{tabular}{|c|c|c|c|c|c|c|c|c|c|c|c|}
\hline $\mathrm{N}^{\circ}$ & Code labo. & Site & Sondage & $\begin{array}{l}\text { Profon- } \\
\text { deur (cm) }\end{array}$ & $\begin{array}{l}\text { Alt. } \\
\text { NGF } \\
\text { (m) }\end{array}$ & $\begin{array}{l}\text { Matériel } \\
\text { daté }\end{array}$ & $\begin{array}{c}\text { Age }{ }^{1+} \mathrm{C} B P \\
\pm 1 \sigma\end{array}$ & $\delta^{12} \mathrm{C}$ & $\begin{array}{c}\text { Dates cali- } \\
\text { brées à } 2 \sigma \\
\text { (Reimer et al. } \\
\text { 2004) }\end{array}$ & $\begin{array}{l}\text { Age Cal. BP } \\
( \pm 2 \sigma) \\
\text { Max. } \\
\text { (médian) } \\
\text { min. }\end{array}$ & $\begin{array}{c}\text { Niveaux } \\
\text { des PMVE } \\
( \pm 0,51 \mathrm{~m})\end{array}$ \\
\hline 1 & Erl-10677 & Porguen & $\mathrm{P}-\mathrm{C} 2$ & $60-70$ & $\begin{array}{l}+2,75 \\
+2,65\end{array}$ & $\begin{array}{c}\text { Limons } \\
\text { organiques }\end{array}$ & $3025 \pm 60$ & $-27,2$ & $\begin{array}{c}1424-1115 \\
\text { cal. BC }\end{array}$ & $\begin{array}{c}3374(3230) \\
3039\end{array}$ & $-0,90$ \\
\hline 2 & Lrl-10678 & Porzguen & $\mathrm{P}-\mathrm{C} 2$ & $340-350$ & $\begin{array}{l}-0,05 ; \\
-0,15 \\
\end{array}$ & $\begin{array}{c}\text { Limons } \\
\text { organiques }\end{array}$ & $3500 \pm 60$ & $-27,2$ & $\begin{array}{c}1975-1687 \\
\text { cal. BC }\end{array}$ & $\begin{array}{c}3921(3770) \\
3635 \\
\end{array}$ & $-3,15$ \\
\hline 3 & Erl-10679 & Porzguen & $\mathrm{P}-\mathrm{C} 2$ & $410-420$ & $\begin{array}{l}-0,75 \\
-0,85\end{array}$ & $\begin{array}{c}\text { Limons } \\
\text { organiques }\end{array}$ & $4280 \pm 60$ & $-27,4$ & $\begin{array}{c}3086 \cdot 2677 \\
\text { cal. BC }\end{array}$ & $\begin{array}{c}5036(4850) \\
4623\end{array}$ & $-3,93$ \\
\hline 4 & E.rl-10680 & Porzguen & $\mathrm{P}-\mathrm{C} 2$ & $470-480$ & $\begin{array}{l}-1,35 ; \\
-1,45\end{array}$ & $\begin{array}{c}\text { Tourbe } \\
\text { (sommet) }\end{array}$ & $4640=60$ & $-28,4$ & $\begin{array}{c}3632-3125 \\
\text { cal. BC }\end{array}$ & $\begin{array}{c}5581(5400) \\
5072\end{array}$ & $-5,15$ \\
\hline 5 & Erl-10681 & Porzguen & $\mathrm{P}-\mathrm{C} 2$ & $500-510$ & $\begin{array}{l}-1,65 \\
-1,75\end{array}$ & $\begin{array}{l}\text { Tourbe } \\
\text { (base) }\end{array}$ & $4775 \pm 60$ & $-28,6$ & $\begin{array}{c}3657-3375 \\
\text { cal. BC }\end{array}$ & $\begin{array}{c}5607(5510) \\
5323\end{array}$ & $-5,49$ \\
\hline 6 & Lrl-10682 & 'Troaon & $\mathrm{T}-\mathrm{C} 2$ & $60-70$ & $\begin{array}{l}+2,80 \\
+2,70\end{array}$ & $\begin{array}{c}\text { Limons } \\
\text { organiques }\end{array}$ & $940 \pm 56$ & $-26,5$ & $\begin{array}{c}\text { cal. AD 997 - } \\
1215\end{array}$ & $\begin{array}{c}952(850) \\
734 \\
\end{array}$ & $-0,22$ \\
\hline 7 & Erl-10683 & Troaon & $\mathrm{T}-\mathrm{C} 2$ & $340-350$ & $\begin{array}{l}0,00 \\
-0,10\end{array}$ & $\begin{array}{c}\text { Limons } \\
\text { organiques }\end{array}$ & $3690 \pm 70$ & $-28,3$ & $\begin{array}{c}2214-1897 \\
\text { cal. BC }\end{array}$ & $\begin{array}{c}4232(4030) \\
3844\end{array}$ & $-2,52$ \\
\hline 8 & Erl-10684 & Troan & $\mathrm{T}-\mathrm{C} 2$ & $380-390$ & $\begin{array}{l}-0,40 \\
-0,50\end{array}$ & $\begin{array}{c}\text { Limons } \\
\text { organiques }\end{array}$ & $4230 \pm 60$ & $-28,8$ & $\begin{array}{c}2927-2590 \\
\text { cal. BC }\end{array}$ & $\begin{array}{c}4947\{4740\} \\
4539\end{array}$ & $-4,21$ \\
\hline 9 & Erl-10685 & Troaon & $\mathrm{T}-\mathrm{C} 2$ & $480-490$ & $\begin{array}{l}-1,40 \\
-1,50\end{array}$ & $\begin{array}{c}\text { Tourbe } \\
\text { (sommet) }\end{array}$ & $4440 \pm 60$ & $-28,7$ & $\begin{array}{c}3339 \cdot 2924 \\
\text { cal. BC }\end{array}$ & $\begin{array}{c}5287(5070) \\
4873\end{array}$ & $-5,04$ \\
\hline 10 & Erl-10686 & Troaon & T-C2 & $510-520$ & $\begin{array}{l}-1,70 \\
-1,80\end{array}$ & $\begin{array}{c}\text { Tourbe } \\
\text { (base) }\end{array}$ & $5450 \pm 70$ & $-28,1$ & $\begin{array}{c}4451-4071 \\
\text { cal. BC }\end{array}$ & $\begin{array}{c}6400(6250) \\
6019\end{array}$ & $-5,02$ \\
\hline 11 & Lrl-11753 & Arun & A-C10 & $90-100$ & $\begin{array}{c}+2,62 ; \\
+2,52\end{array}$ & $\begin{array}{l}\text { Charbon } \\
\text { de bois }\end{array}$ & $1081 \pm 56$ & $-24,1$ & $\begin{array}{c}\text { cal. AD } 780 \text { - } \\
1034\end{array}$ & $\begin{array}{c}1170(1000) \\
914\end{array}$ & $-1,05$ \\
\hline 12 & Erl-11754 & Arun & $\mathrm{A}-\mathrm{C} 10$ & $195-200$ & $\begin{array}{l}+1,57 \\
+1,52\end{array}$ & $\begin{array}{c}\text { Limons } \\
\text { organiques }\end{array}$ & $3277 \pm 59$ & $-26,1$ & $\begin{array}{c}1687-1435 \\
\text { cal. BC }\end{array}$ & $\begin{array}{c}3637(3510) \\
3383 \\
\end{array}$ & - \\
\hline 13 & Erl-11749 & Arun & $\mathrm{A}-\mathrm{Cl} 4$ & $40-50$ & $\begin{array}{l}+3,10 ; \\
+3,00\end{array}$ & $\begin{array}{c}\text { Limons } \\
\text { organiques }\end{array}$ & $-436 \pm 55$ & $-24,3$ & $\begin{array}{c}\text { cal. AD 1956- } \\
2000\end{array}$ & - & $-0,41$ \\
\hline 14 & Erl-11750 & Arun & A.Cl4 & $140-150$ & $\begin{array}{l}+2,10 \\
+2,00\end{array}$ & $\begin{array}{c}\text { Limons } \\
\text { organiques }\end{array}$ & $1686 \pm 56$ & $-25,8$ & $\begin{array}{l}\text { cal. } A D 234- \\
533\end{array}$ & $\begin{array}{c}1716(1600) \\
1417 \\
\end{array}$ & $-0,90$ \\
\hline 15 & Erl-11751 & Arun & $\mathrm{A}-\mathrm{Cl} 4$ & $270-280$ & $\begin{array}{l}+0,80 ; \\
+0,70\end{array}$ & $\begin{array}{l}\text { Charbon } \\
\text { de bois }\end{array}$ & $2340 \pm 54$ & $-26,6$ & $\begin{array}{c}744-210 \text { cal. } \\
\text { BC }\end{array}$ & $\begin{array}{c}2693(2370) \\
2158\end{array}$ & $-1,97$ \\
\hline 16 & Erl-11752 & Arun & $\mathrm{A}-\mathrm{Cl} 4$ & $360-370$ & $\begin{array}{l}-0,10 \\
-0,20\end{array}$ & $\begin{array}{l}\text { Charbon } \\
\text { de bois }\end{array}$ & $2716 \pm 55$ & $-27,2$ & $\begin{array}{c}996-798 \mathrm{cal} . \\
\text { BC }\end{array}$ & $\begin{array}{c}2943(2820) \\
2747\end{array}$ & $-3,35$ \\
\hline
\end{tabular}

L'unité 2 est essentiellement composée d'une vase grise argilo-silteuse (grain moyen compris entre 20 et $40 \mu \mathrm{m}$ ), contenant de nombreuses radicelles de végétation halophile. L'épaisseur de cette formation atteint $4,5 \mathrm{~m}$ au niveau du sondage T-C1 et décroît vers l'intérieur du marais maritime (fig. 5A). Les datations radiocarbone obtenues le long du sondage T-C2 s'étalent du Subboréal au Subatlantique, entre 4740 
cal.BP et 850 cal.BP, indiquant une mise en place dans un contexte de transgression marine continue. Dans l'ensemble, la composition en foraminifères est relativement homogène. Les assemblages sont dominés par Trochammina inflata et Jadammina macrescens (fig. 6). La fonction de transfert établit en moyenne un niveau de sédimentation situé à 2,7 $\pm 0,53 \mathrm{~m}$ au-dessus du niveau moyen de la mer, ce qui correspond à l'étage occupé par le haut schorre.

Figure 5 : Séquences stratigraphiques longitudinales des marais de Troaon (A), Porzguen (B) et Arun (C)/Sediment sequences across Troaon (A), Porzguen (B) and Arun salt marshes

A) TROAON

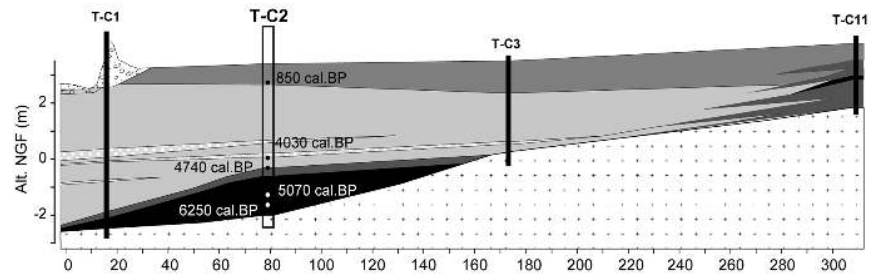

B) PORZGUEN
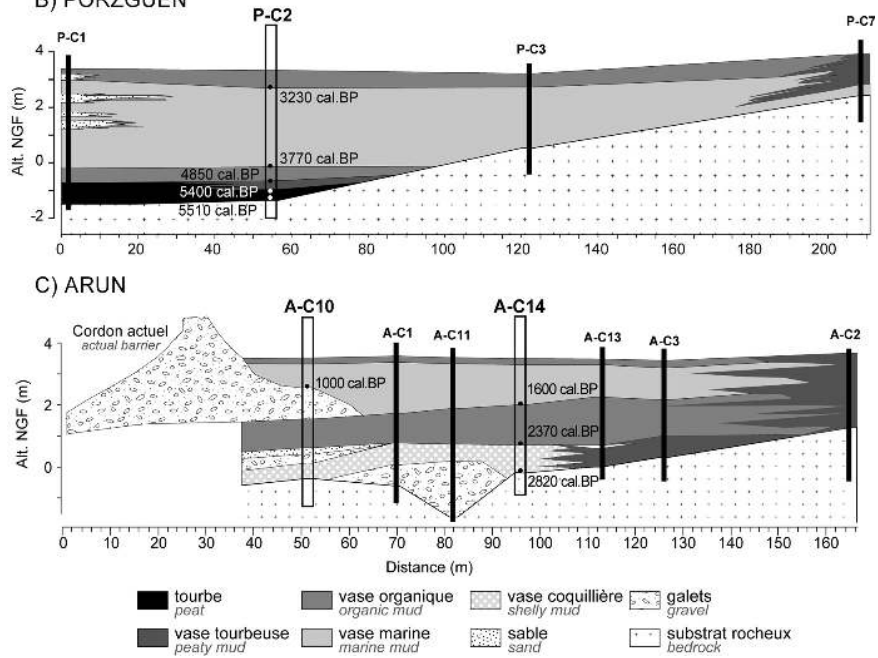

$\mathrm{Au}$ sein de cette unité, deux passées sablo-vaseuses très riches en coquilles d'hydrobies et de coques sont identifiées (unités $2 \mathrm{~B}$ et $2 \mathrm{D}$ ). La granulométrie est plus grossière et le cortège de foraminifères associé est également différent du reste de l'unité 2 . Les espèces carbonatées telles que Haynesina germanica et Elphidium spp. dominent (fig. 6). Ce type d'assemblage est caractéristique du bas schorre, voire de la haute slikke. La fonction de transfert fixe donc le niveau de sédimentation entre $0,6 \pm 0,51 \mathrm{~m}$ et 1,3 $\pm 0,53 \mathrm{~m}$ au-dessus du niveau moyen de la mer. L'application de la technique MAT souligne toutefois l'absence d'équivalents actuels associés à ces assemblages. Ces deux niveaux de vase coquillière ne doivent pas être interprétés comme les témoins de pulsations transgressives, mais sont plutôt à considérer comme des dépôts de tempêtes, associés à la destruction brutale et à la reformation d'un cordon littoral protégeant auparavant le marais maritime. L'ouverture de brèches dans une accumulation située plus en avant sur l'estran a probablement entrainé le transfert rapide, vers l'intérieur du marais, de sédiments fins situés sur le bas de plage. Ce transfert a favorisé un certain brassage des populations de foraminifères occupant les différents étages littoraux, ce qui explique, d'une part, la forte proportion des espèces carbonatées dans les échantillons et, d'autre part, l'absence d'analogues modernes permettant de déterminer la position altitudinale de ces assemblages fossiles. 
Figure 6 : Lithostratigraphie et biostratigraphie (foraminifères) du sondage T-C2 (marais de Troaon)/Lithostratigraphy and biostratigraphy (foraminifera): borehole T-C2 (Troaon salt marsh)

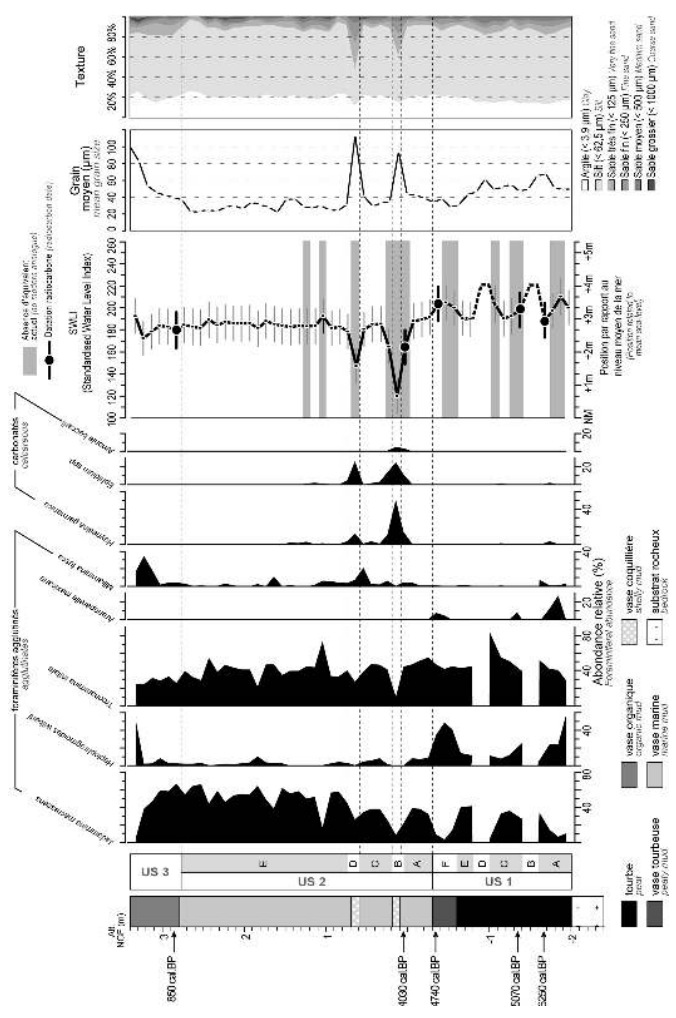

L'unité 3 correspond à un dépôt argilo-silteux contenant une fraction organique importante, essentiellement constituée de radicelles en cours d'oxydation. Ce dépôt présente également un enrichissement en sable et une granulométrie croissante à mesure que l'on atteint la surface (fig. 6), en raison d'une probable modification des caractéristiques hydrodynamiques du marais maritime. Cette unité montre une épaisseur comprise en 0,6 et 1,2 m (fig. 5A). La datation effectuée à la base de cette unité indique une mise en place au cours du dernier millénaire de notre ère. La composition en foraminifères est assez semblable à celle de l'unité 2 . Elle est dominée par Trochammina inflata et Jadammina macrescens et permet d'estimer le niveau de sédimentation à 2,8 $\pm 0,52 \mathrm{~m}$ au-dessus du niveau moyen de la mer (fig. 6).

\section{Le marais de Porzguen : description de la stratigraphie et des unités stratigraphiques}

À l'instar du marais de Troaon, la séquence stratigraphique du marais de Porzguen est transgressive (fig. 5B). Trois unités stratigraphiques peuvent également être distinguées à la suite de l'analyse sédimentologique et de l'étude de la micro-faune (fig. 7).

L'unité 1 repose sur des argiles issues de l'altération des schistes dévoniens (formation des schistes de Porzguen) constituant le substratum rocheux. D'une épaisseur de $1 \mathrm{~m}$ en moyenne, le dépôt argilo-silteux comporte une importante fraction organique et contient de nombreux macro-restes végétaux (fragments de bois, feuilles et radicelles). Cette unité stratigraphique est située à une altitude comprise entre $-1,65 \mathrm{~m}$ et $-0,65 \mathrm{~m}$ 
NGF. Les datations radiocarbone livrent des âges compris entre 5510 cal.BP et 4850 cal.BP et permettent de situer la mise en place de ce dépôt durant le Subboréal. Dans le détail, deux sous-unités peuvent être distinguées (fig. 7). La base de l'unité 1 (unité 1A) correspond à une formation de tourbière littorale, dont la mise en place s'échelonne entre 5510 cal.BP et 5400 cal.BP. Les assemblages de foraminifères sont dominés par Trochammina inflata et Haplophragmoides wilberti. Bien que certains échantillons n'aient pas d'équivalent actuel, la fonction de transfert établit le niveau de sédimentation à $+3,4 \pm 0,55 \mathrm{~m}$ par rapport au niveau moyen de la mer. L'unité $1 \mathrm{~B}$ est composée d'une vase tourbeuse et se distingue de l'unité $1 \mathrm{~A}$ d'un point de vue granulométrique par un léger enrichissement en argile et un grain moyen situé autour de $40 \mu \mathrm{m}$. L'âge de ce dépôt est compris entre 5400 cal.BP et 4850 cal.BP. Les assemblages de foraminifères montrent une part plus importante de Jadammina macrescens aux dépens d'Haplophragmoides wilberti suggérant un niveau de sédimentation compris entre $+2,8$ et $+3 \pm 0,54 \mathrm{~m}$ par rapport au niveau marin moyen. Cette position altitudinale correspond à la partie inférieure du haut schorre.

31 L'unité 2 est constituée d'une vase marine argilo-silteuse (fig. 7), plus organique à la base, dont l'épaisseur atteint 3,30 m au niveau du sondage P-C2 (fig. 5B) et dont la mise en place se situe durant le Subboréal. Les datations livrent des âges compris entre 4850 cal.BP et 3230 cal.BP. Les niveaux sableux rencontrés par le sondage Porz-C1 sont liés à la divagation du chenal principal de marée drainant le marais. La composition en foraminifères permet de distinguer deux sous-unités $2 \mathrm{~A}$ et $2 \mathrm{~B}$. La partie inférieure de l'unité stratigraphique 2 (unité 2A) est dominée par Trochammina inflata et Jadammina macrescens et indique un niveau de sédimentation correspondant à la partie inférieure du haut schorre. En revanche, la partie supérieure de l'unité 2 (unité 2B) se caractérise par une diminution importante de Jadammina macrescens et la prédominance de Trochammina inflata dans les assemblages fossiles. Son abondance relative dépasse $90 \%$ dans certains échantillons. Une telle proportion de Trochammina inflata n'est jamais retrouvée dans les échantillons prélevés à la surface des marais de Lanveur et d'Arun (figure 4). Ce type d'assemblage n'a donc aucun équivalent actuel et la fonction de transfert ne peut déterminer le niveau de sédimentation de façon satisfaisante. La raison qui explique cette surreprésentation de Trochammina inflata doit être recherchée dans les processus taphonomiques qui affectent les tests de foraminifères après leur mort. Avec une taille plus importante et un test souvent plus épais, Trochammina inflata résiste mieux à la fragmentation post-mortem et se retrouve en plus grande proportion dans les échantillons fossiles. Cette abondance nous livre néanmoins une information qualitative sur le niveau de sédimentation puisque Trochammina inflata est dominante dans les parties hautes du schorre, en limite avec les tourbières littorales occupées par les roselières à phragmites. Par conséquent, l'unité $2 \mathrm{~B}$ peut être associée à un dépôt de haut schorre, voire de tourbière. En outre, l'absence totale de foraminifères à deux niveaux de l'unité $2 \mathrm{~B}$ pourrait indiquer une continentalisation du milieu. 
Figure 7 : Lithostratigraphie et biostratigraphie (foraminifères) du sondage P-C2 (marais de Porzguen)/Lithostratigraphy and biostratigraphy (foraminifera): borehole P-C2 (Porzguen salt marsh)

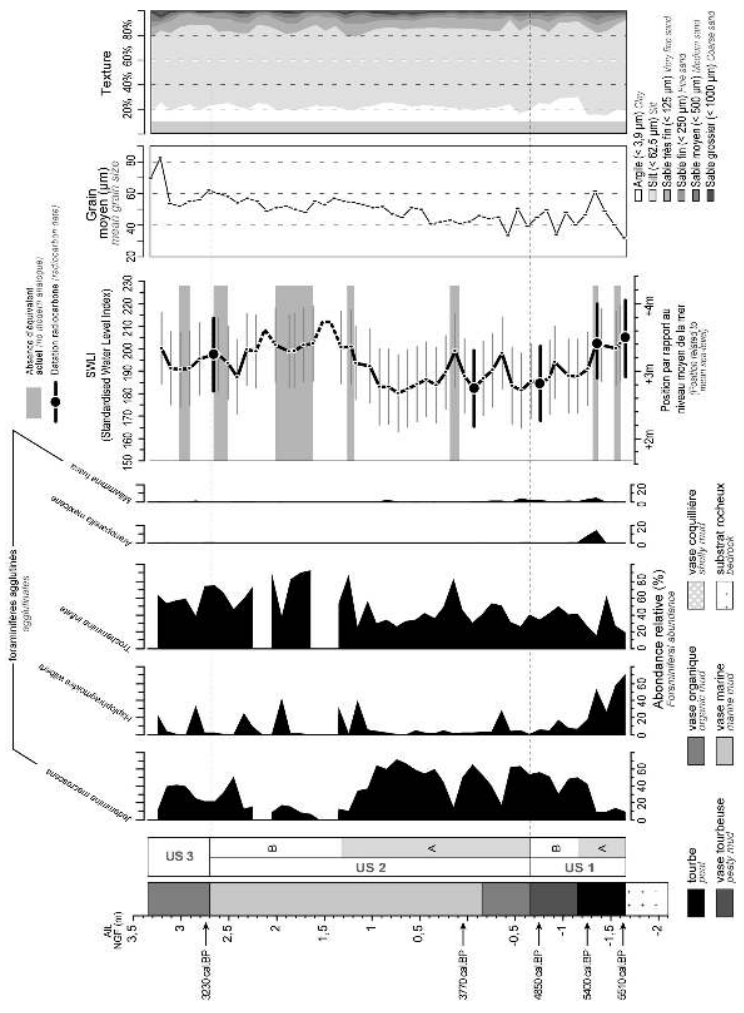

L'unité 3 se compose d'une vase organique argilo-silteuse (fig. 7), très tassée, dont la mise en place est associée à un atterrissement progressif du marais, dans un contexte de stabilité relative du niveau marin tardi-holocène. La base de cette unité livre un âge de 3230 cal.BP.

\section{Le marais d'Arun : description de la stratigraphie et des unités stratigraphiques}

La séquence stratigraphique relevée dans le marais maritime d'Arun se compose de six unités bien différenciées, dont la mise en place est associée à plusieurs phases de construction/destruction de cordons littoraux, vraisemblablement dans un contexte de transgression marine (fig. 5C).

L'unité 1 se compose d'un matériel sablo-graveleux reposant entièrement sur le substratum rocheux (schistes dévoniens) dont elle comble une légère dépression topographique. L'épaisseur de ce dépôt atteint $1,5 \mathrm{~m}$ au niveau du sondage A-C11 et décroît vers le sondage A-C1, avant de disparaitre totalement (fig. 5C). La nature et l'épaisseur du matériel sédimentaire nous incitent à considérer cette unité stratigraphique comme le témoin d'un ancien cordon étalé dont la mise en place serait associée à une phase de transgression marine ayant amenée le niveau de la mer à un niveau proche de l'actuel vers 3000 BP. Ainsi s'explique la position interne de ce dépôt par rapport au cordon actuel.

L'unité 2 est constituée d'un matériel sablo-vaseux (fig. 8 et 9) comportant de nombreux débris coquilliers ainsi que des macro-restes végétaux. Le grain moyen est 
d'environ $200 \mu \mathrm{m}$ et indique une sédimentation en milieu relativement ouvert sur le large. Au niveau du sondage $\mathrm{A}-\mathrm{C} 14$, la datation obtenue à la base de cette unité donne un âge de 2820 cal.BP et fixe la mise en place de ce dépôt à la transition Subboréal/ Subatlantique. Les assemblages de foraminifères sont dominés par Trochammina inflata et Jadammina macrescens et sont caractéristiques de la partie supérieure du haut schorre (fig. 8). Le niveau de sédimentation est situé autour de $+2,8 \pm 0,53 \mathrm{~m}$ par rapport au niveau moyen de la mer et indique que cette unité correspond à un dépôt de haut d'estran.

L'unité 3 se compose d'un matériel sablo-graveleux à la base et sableux au sommet, sur une épaisseur totale de $0,5 \mathrm{~m}$. Ce niveau ne contient aucun foraminifère (fig. 8) et correspond à un dépôt d'overwash affectant un cordon littoral situé dans une position similaire à la flèche actuelle. Cet épisode érosif, probablement lié à une succession d'épisodes tempétueux, n'a pas été daté précisément. Compte tenu de sa position stratigraphique, nous pouvons néanmoins proposer un âge compris entre 2800 cal.BP et 2370 cal.BP, correspondant à la transition Subboréal/Subatlantique.

L'unité 4 correspond à une vase argilo-silteuse (grain moyen compris entre $30 \mu \mathrm{m}$ et $50 \mu \mathrm{m}$ ), très organique, contenant de nombreuses radicelles de plantes halophiles. Son épaisseur varie entre $1,3 \mathrm{~m}$ et $0,7 \mathrm{~m}$. Sa mise en place s'échelonne entre $2370 \mathrm{cal} . \mathrm{BP}$ et 1600 cal.BP. Les assemblages de foraminifères fossiles sont dominés par Trochammina inflata et Jadammina macrescens, indiquant un niveau de sédimentation de haut schorre. Sur le sondage Arun-C14, l'abondance de Trochammina inflata suppose toutefois un niveau de sédimentation plus élevé au sommet qui pourrait être le résultat d'une légère régression marine datant de l'Âge du Bronze et se terminant à l'époque gallo-romaine, comme le suggère M.-Th. Morzadec-Kerfourn (1969).

Figure 8 : Lithostratigraphie et biostratigraphie (foraminifères) du sondage A-C14 (marais d'Arun)/ Lithostratigraphy and biostratigraphy (foraminifera): borehole A-C14 (Arun salt marsh)

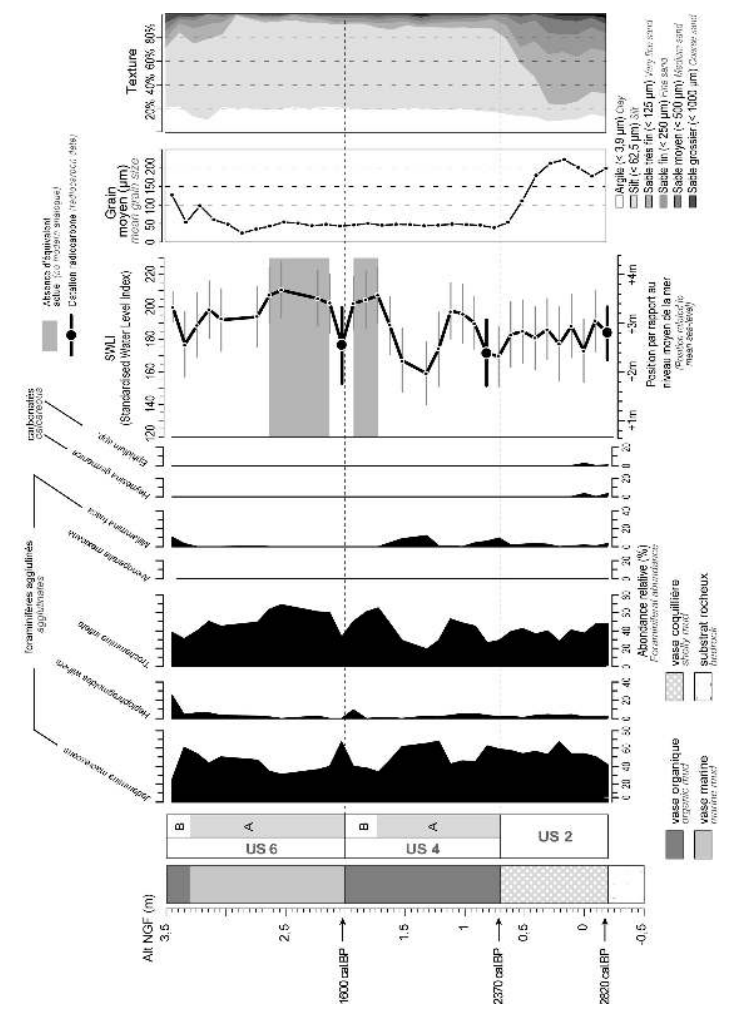


Figure 9 : Lithostratigraphie et biostratigraphie (foraminifères) du sondage A-C10 (marais d'Arun)/ Lithostratigraphy and biostratigraphy (foraminifera): borehole A-C10 (Arun salt marsh)

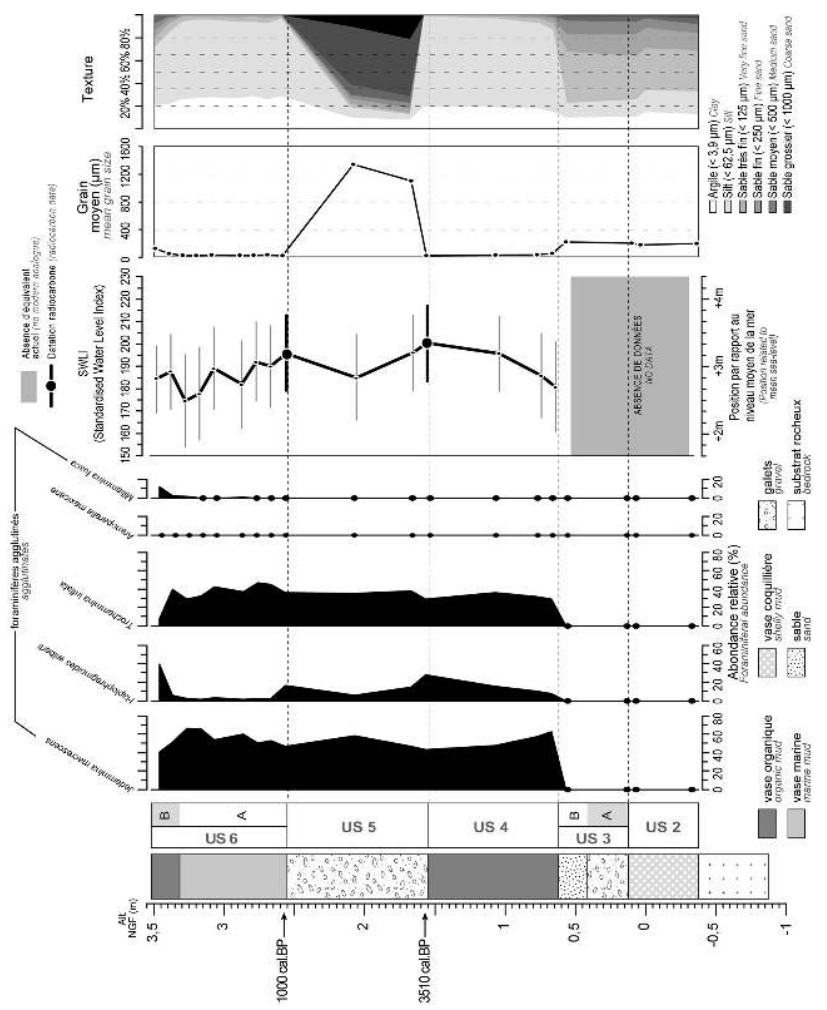

L'unité 5 se rencontre uniquement sur le sondage A-C10 (fig. 9) où elle forme un dépôt sablo-graveleux (grain moyen supérieur à $1 \mathrm{~mm}$ ) d'une épaisseur d'un mètre environ. À l'exutoire du chenal de marée, ce dépôt est également visible en coupe sur l'une des berges sapée en microfalaise. Compte tenu de son étendue, sa mise en place ne doit pas être associée à un événement d'overwash localisé, mais plutôt à une phase érosive plus générale impliquant le recul et l'écrêtement complet d'un cordon littoral. Ce cordon devait former une flèche assez identique à la flèche actuelle, qui aurait contraint le chenal de vidange du marais à décrire un méandre pour rejoindre la mer.

La mise en place de l'unité 6 débute vers 1600 cal.BP par une sédimentation argilosilteuse (grain moyen d'environ $50 \mu \mathrm{m}$ ). Cette vase contient également une fraction organique importante. Elle représente la phase finale du remplissage sédimentaire du marais maritime d'Arun. La partie sommitale de cette unité est plus organique (unité $6 \mathrm{~B}$ ) et contient de nombreux débris végétaux en voie de décomposition. Les assemblages de foraminifères sont dominés par Trochammina inflata et Jadammina macrescens caractéristiques d'un environnement de haut schorre (fig. 8 et 9). La fonction de transfert détermine le niveau de sédimentation à $+3 \pm 0,57 \mathrm{~m}$ par rapport au niveau moyen de la mer.

\section{Courbes des variations relatives du niveau marin}

Le long du sondage P-C2, cinq datations radiocarbone ont permis de caler dans le temps les fluctuations du niveau de la mer estimées par l'application de la fonction de transfert aux assemblages de foraminifères fossiles (fig. 10A). Les assemblages présents dans le niveau de tourbe littorale, à la base du sondage, indiquent que, lors de son 
dépôt, le sédiment se situait à $3,46 \pm 0,56 \mathrm{~m}$ au-dessus du niveau moyen de la mer. Or, cet échantillon est désormais situé à une altitude de -1,64 m NGF, soit 2,02 m sous le niveau moyen de la mer actuel en rade de Brest. Ces données permettent donc de déterminer l'ancienne position du niveau marin à $-5,5 \mathrm{~m}(3,46 \mathrm{~m}+2,02 \mathrm{~m})$ par rapport à l'actuel aux alentours de 5500 cal.BP.

41 À partir de cette date et jusqu'à 3200 cal.BP, la remontée du niveau marin s'effectue rapidement, à une vitesse moyenne de $2 \mathrm{~mm} / \mathrm{an}$. Le niveau marin atteint alors une position voisine de l'actuelle, située à $-0,9 \mathrm{~m}$ par rapport à aujourd'hui. Après $3200 \mathrm{cal}$. $\mathrm{BP}$, les données recueillies dans ce secteur sont insuffisantes pour déterminer les variations du niveau marin.

Le long du sondage T-C2, les niveaux tourbeux de base ont permis de déterminer la position relative du niveau marin à $5 \mathrm{~m}$ en dessous de sa position actuelle entre 6250 cal.BP et 5070 cal.BP, ce qui semble indiquer un certain stationnement du niveau marin durant cette période. Cette phase de stabilité est suivie d'une remontée rapide du niveau marin entre 5070 et 4030 cal.BP (fig. 10B), à une vitesse de $2,4 \mathrm{~mm} / \mathrm{an}$. La courbe se caractérise ensuite par deux oscillations de forte amplitude, amenant le niveau marin relatif à un niveau proche de l'actuel. Ces deux pics correspondent à des dépôts de tempête, probablement associés à une phase de rupture du cordon protégeant initialement le marais de Troaon. À partir de 3200 cal.BP, le niveau marin connaît une transgression régulière qui se poursuit à un rythme d'environ $1 \mathrm{~mm} / \mathrm{an}$ jusqu'à 850 cal.BP, date à laquelle il atteint alors une position voisine de l'actuelle (fig. 10B).

La courbe déduite du sondage A-C14 débute vers 2820 cal.BP et établit la position du niveau marin aux alentours de $-3,3 \mathrm{~m}$ par rapport à l'actuel (fig. 10C). Une série d'oscillations mineures marquent ensuite la transgression marine et doivent également être attribuées aux phases de destructions successives du cordon littoral protégeant le marais d'Arun. Si l'on omet ces petites fluctuations, la courbe illustre un mouvement transgressif assez régulier jusqu'à aujourd'hui. Le niveau marin s'élève progressivement à une vitesse moyenne de $1,2 \mathrm{~mm} / \mathrm{an}$. Les résultats obtenus pour le sondage $\mathrm{A}-\mathrm{C} 10$ suggèrent une tendance similaire pour le dernier millénaire (fig. 10D). 
Figure 10 : Courbes de remontée du niveau de la mer basées sur les datations radiocarbone et sur l'analyse des foraminifères présents dans les sondages de référence P-C2 (A), T-C2 (B), A-C14 (C) et A-C10 (D)/Relative sea-level curves and index points obtained using foraminiferal-based transfer function: borehole P-C2 (A), T-C2 (B), A-C14 (C) and A-C10 (D)
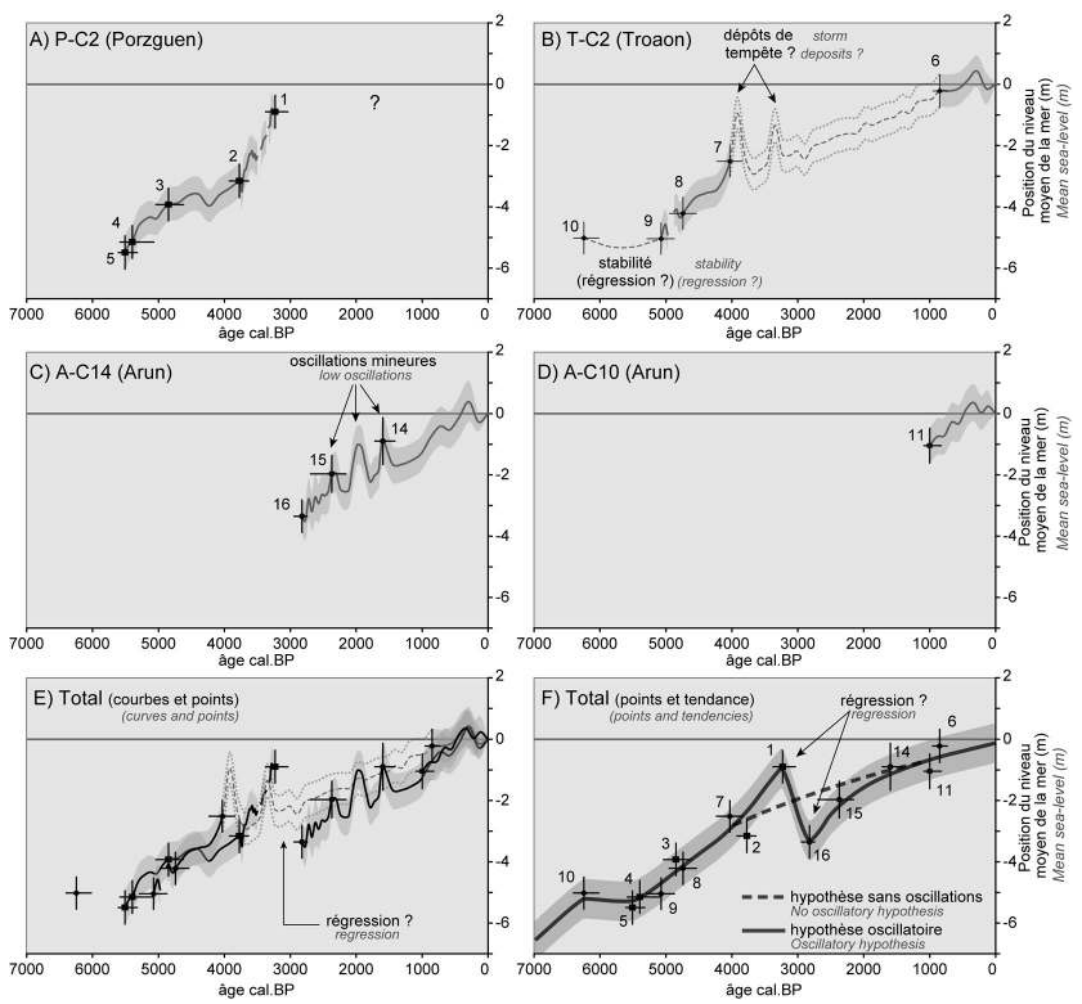

E. Superposition des quatre courbes soulignant les différences importantes dans les résultats obtenus d'un site à un autre. F. Courbe dressée uniquement d'après le positionnement des niveaux datés au ${ }^{14} \mathrm{C}$

Superposition of the four curves (E) and sea-level curve only based on sea-level index points (F).

La superposition des quatre courbes de remontée relative du niveau marin, obtenues à partir des sondages de référence, révèle des écarts importants dans les reconstitutions basées sur l'utilisation de la fonction de transfert (fig. 10E). Les plus grandes différences concernent les tendances enregistrées au cours des 3000 dernières années. En effet, vers 3000 cal.BP, la courbe dressée à partir du sondage P-C2 estime la position du niveau marin au voisinage de l'actuel, tandis que les courbes obtenues sur Troaon et Arun la situe $3 \mathrm{~m}$ en dessous. Dans le premier cas, le niveau de la mer aurait connu une relative stabilité durant les trois derniers millénaires. Dans les autres cas, il aurait enregistré un mouvement transgressif régulier jusqu'à aujourd'hui, à une vitesse moyenne de $1 \mathrm{~mm} / \mathrm{an}$. L'hypothèse permettant de concilier ces deux cas de figure fait intervenir une légère régression marine entre 3200 et 2800 cal.BP (fig. 10F).

\section{Discussion}

\section{Erreurs et incertitudes}

La première source d'incertitude concerne le fait que la fonction de transfert n'a trouvé aucun équivalent actuel pour une trentaine d'échantillons analysés, dont trois ont été datés au radiocarbone $\left(n^{\circ} 4,7,9\right.$, fig. 4$)$ et ont servi à construire notre courbe de 
remontée du niveau marin. Les lacunes de ce type sont fréquemment rencontrées dans les travaux utilisant les fonctions de transfert comme outils pour déterminer les anciennes positions du niveau marin relatif (Edwards et Horton, 2000; Horton et Edwards, 2006). L'absence d'équivalent actuel tient généralement aux processus taphonomiques affectant les tests de foraminifères après leur mort (fragmentation, dissolution). Pour ces échantillons, le positionnement altitudinal du niveau marin est seulement approximatif, le modèle statistique s'appuyant sur des assemblages fossiles incomplets (Massey et al., 2006).

La seconde source d'erreur concerne le tassement des sédiments après leur dépôt qui n'a pas été pris en compte dans cette étude. Bien que cette question soit fréquemment abordée par les auteurs (Pirazzoli, 1976 ; Cahoon et al., 1995 ; Vella, 1999, 2004 ; Vella et Provansal, 2000; Long et al., 2006; Edwards, 2006), il n'existe pas, à ce jour, de méthodologie commune à l'ensemble de la communauté scientifique pour corriger ce type d'erreur (Massey et al., 2008). Cela tient en grande partie au manque de modèle géotechnique capable d'intégrer la compaction des dépôts au sein de stratigraphies complexes. Toutefois, en rade de Brest, les niveaux tourbeux rencontrés à la base des stratigraphies comprennent une fraction minérale importante (environ $30 \%$ ) qui atténue la compaction et limite les erreurs potentielles de positionnement du niveau marin. En outre, une grande majorité des datations a été effectuée sur des niveaux de vase peu compressibles.

Enfin, des erreurs éventuelles de datations ne peuvent pas être totalement exclues et ont pu nous conduire à un mauvais positionnement dans le temps de quelques niveaux marins. En effet, plusieurs datations ont été réalisées sur la matière organique totale présente dans les échantillons. Or, ces échantillons ont pu contenir du carbone de pollution, plus récent ou plus ancien, dont la présence est due à des phénomènes d'imprégnation ou de redéposition (Delibrias et Giot, 1970). Comme le rappelle J. Evin (1986), le carbone de pollution a le même état chimique que celui de l'origine et ne peut donc être éliminé en toute certitude par le biais de traitement en laboratoire. Par conséquent, les résultats doivent être interprétés avec la plus grande précaution.

\section{Comparaison régionale (avec la courbe de Morzadec-Kerfourn)}

Dans la figure 11, la courbe dressée en rade de Brest est comparée avec celle de M.Th. Morzadec-Kerfourn (1974) obtenue pour le nord du Finistère dont les dates ont été corrigées par calibration (tableau 2) au moyen du logiciel Calib 6.0, selon la même méthode que celle décrite précédemment. Ces courbes se réfèrent au niveau des pleines mers de vive-eau (PMVE) actuelles. Les deux courbes présentent grossièrement la même allure. La remontée du niveau marin depuis 6500 cal.BP se décline en quatre étapes.

Tout d'abord, entre 6500 BP et 5000 cal.BP, le niveau de la mer montre une relative stabilité. La courbe de la rade de Brest estime cependant le niveau des pleines mers de vive-eau autour de $-5,5 \mathrm{~m}$ sous son niveau actuel, tandis que la courbe dressée dans le nord du Finistère envisage un stationnement à $-8,8 \pm 1 \mathrm{~m}$. Cet écart résulte vraisemblablement d'un tassement plus important des niveaux tourbeux étudiés par M.-Th. Morzadec-Kerfourn, dont l'épaisseur atteint parfois $1 \mathrm{~m}$. Dans le nord Finistère, ces niveaux affleurent désormais sur l'estran, en avant de cordons dunaires qui les ont transgressés. Ces dépôts ont donc subi un effet de compaction important lors du recul 
vers l'intérieur des terres des cordons littoraux. Les habitats côtiers du Néolithique, comme ceux du Curnic à Guisseny, qui sont aujourd'hui submergés puisqu'ils se situent au niveau actuel de mi-marée, correspondent à cette période de bas niveau marin. Ils apparaissent désormais sur les estrans à la faveur de fortes marées ou de tempêtes, et indiquent que durant toute la période néolithique, la mer s'est maintenue à un niveau bien inférieur à l'actuel. De nombreux monuments mégalithiques ont également été construits à cette époque, vraisemblablement hors d'atteinte de la mer. Le menhir de Men Ozac'h en Plouguerneau a été élevé entre 5000 et 4500 cal.BP. Or, sa base se trouve actuellement à $6,4 \mathrm{~m}$ au-dessous des plus hautes mers actuelles. De nombreux témoins archéologiques, contemporains de cette phase de relative stabilité du niveau de la mer, existent également dans le golfe du Morbihan (cercle de menhirs d'ErLannig) et dans le sud du Finistère (menhir de Pen-Laouig dans la rivière de Pontl'Abbé, menhir de Lesconil à Plobannalec, dolmen d'Ezer à Loctudy) (Le Gouletquer et Kayser, 1992 ; Giot, 1992). Enfin, la tourbe dulçaquicole retrouvée sur la plage de Gwen Trez à Argenton affleure actuellement entre 4 et $5 \mathrm{~m}$ sous les plus hautes mers. Ce dépôt est daté de 4430 cal.BP et précède immédiatement un niveau de tourbe littorale témoignant d'une reprise de la transgression (tableau 2). Notons, par ailleurs, que cette phase de stabilité relative du niveau marin a également été mise en évidence dans le nord de la France (bassin de Saint-Omer) par E. Gandouin et al. (2007).

Figure 11 : Comparaison des courbes des variations du niveau marin relatif dressées en rade de Brest et dans le nord Finistère (les datations marquées par des lettres sont référencées dans le tableau 2)/Comparison between relative sea-level curves obtained in the Bay of Brest and in the north-western part of Brittany (dates with letters are detailed in table 2)

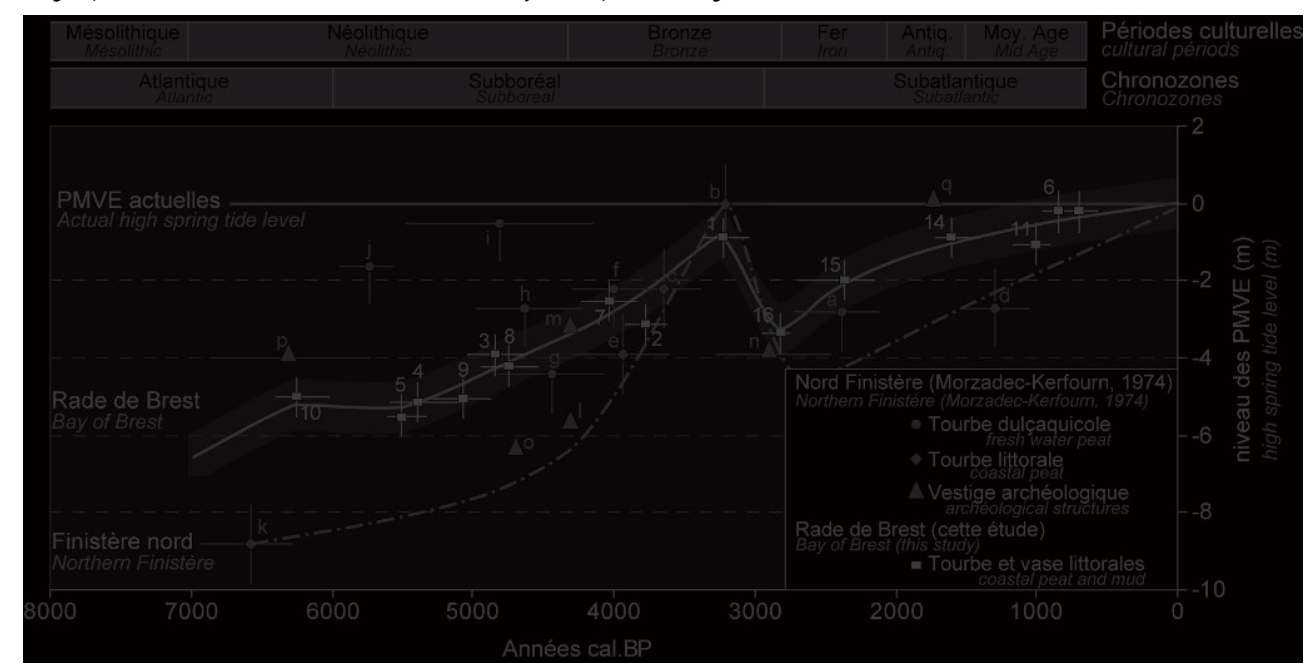

51 Ensuite, entre 4500 et 3200 cal.BP, le niveau marin connaît une transgression relativement plus rapide, estimée à $2 \mathrm{~mm} / \mathrm{an}$ en rade de Brest. Les vitesses de remontée proposées par M.-Th. Morzadec-Kerfourn (1974) sont supérieures (environ $4 \mathrm{~mm} / \mathrm{an}$ ), dans la mesure où l'auteur fait démarrer la transgression quelques mètres en dessous du niveau estimé en rade de Brest. La mer recouvre les sites et les tourbières néolithiques. Des cordons littoraux viennent fermer de petites dépressions côtières et assurent la mise en place de nouvelles tourbières à l'abri de l'agitation marine. Le niveau de tourbe littorale retrouvé à Lampaul-Plouarzel se situe au voisinage des plus hautes mers actuelles et tend à prouver que la mer a atteint, à la fin du Subboréal, un niveau proche de l'actuel vers 3200 cal.BP (fig. 11). Par ailleurs, cette phase 
transgressive est également reconnue dans le sud de la Bretagne, entre la presqu'île de Rhuys et la rivière d'Étel par L. Visset et J. Bernard (2006).

Puis, entre 3200 et 2800 cal.BP, les deux courbes envisagent une légère régression marine relative. Pour M.-Th. Morzadec-Kerfourn (1974), la baisse du niveau marin aurait pu atteindre $4 \mathrm{~m}$ durant cette période. Les données acquises en rade de Brest suggèrent cependant une phase régressive d'amplitude plus modeste d'environ $2 \mathrm{~m}$. Notons que ces résultats apparaissent en contradiction avec le mouvement transgressif enregistré durant cette période sur l'île d'oléron (Clavé et al., 2001) et dans l'estuaire de la Gironde (Pontee et al., 1998). Ils seront discutés plus en détail dans le point suivant de notre discussion.

Enfin, à partir de 2800 cal.BP, les données acquises en rade de Brest et en Finistère nord indiquent une reprise de la transgression marine à un rythme de $1 \mathrm{~mm} / \mathrm{an}$ en moyenne jusqu'à aujourd'hui. Cette remontée lente du niveau marin au cours des derniers millénaires est attestée par la plupart des études effectuées sur la façade atlantique française. Seuls R. L. Jones et al. (1987) font intervenir une baisse du niveau de la mer sur l'île de Jersey entre 1500 cal.BP et aujourd'hui, qu'ils attribuent toutefois à des effets de soulèvement et d'affaissement locaux.

Tableau 2 : Datations radiocarbone des tourbes d'estran et de quelques témoins archéologiques du nord Finistère permettant le positionnement des PMVE/Radiocarbon data obtained in the northwestern part of Brittany

\begin{tabular}{|c|c|c|c|c|c|c|c|c|}
\hline $\mathrm{N}^{\circ}$ & Site & Matériel & $\begin{array}{l}\text { Code } \\
\text { labo. }\end{array}$ & $\begin{array}{l}\text { Age } \\
14 \mathrm{C} \\
\mathrm{BP} \quad \pm \\
1 \mathrm{~s}\end{array}$ & $\begin{array}{l}\text { Age cal. BP } \\
\pm 2 \mathrm{~s} \\
\text { min. } \\
\text { (med).max }\end{array}$ & $\begin{array}{l}\text { Alt. } \\
\text { NGF } \\
\text { (m) }\end{array}$ & $\begin{array}{l}\text { Niveau } \\
\text { des } \\
\text { PMVE } \\
(\mathrm{m})\end{array}$ & Source \\
\hline$a$ & Santec & $\begin{array}{l}\text { tourbe } \\
\text { dulçaquicole }\end{array}$ & $\begin{array}{l}\text { Gif } \\
818\end{array}$ & $\begin{array}{l}2330 \pm \\
105\end{array}$ & $\begin{array}{l}2122(2378) \\
2716\end{array}$ & 2 & $<-2,8 \pm 1$ & $\begin{array}{l}\text { M.-K. } \\
(1969)\end{array}$ \\
\hline$b$ & $\begin{array}{l}\text { Lampaul- } \\
\text { Plouarzel }\end{array}$ & tourbe littorale & $\begin{array}{l}\text { Gif } \\
712\end{array}$ & $\begin{array}{l}3020 \pm \\
110\end{array}$ & $\begin{array}{l}2923(3200) \\
3447\end{array}$ & 3,5 & $0 \pm 1$ & $\begin{array}{l}\text { M.-K. } \\
(1969)\end{array}$ \\
\hline c & $\begin{array}{l}\text { Plouescat } \\
\text { (sommet) }\end{array}$ & tourbe littorale & $\begin{array}{l}\text { Gif } \\
710\end{array}$ & $\begin{array}{l}3390 \pm \\
120\end{array}$ & $\begin{array}{l}3380(3646) \\
3926\end{array}$ & 2,5 & $-2,2 \pm 1$ & $\begin{array}{l}\text { M.-K. } \\
(1969)\end{array}$ \\
\hline d & Plounéour-Trez & tourbe littorale & $\begin{array}{l}\text { Gif } \\
1300\end{array}$ & $\begin{array}{l}1390 \pm \\
120\end{array}$ & $\begin{array}{l}1055(1303) \\
1540\end{array}$ & 2 & $-2,7 \pm 1$ & $\begin{array}{l}\text { M.-K. } \\
(1969)\end{array}$ \\
\hline $\mathrm{e}$ & Argenton (B) & tourbe littorale & $\begin{array}{l}\text { Gif } \\
815\end{array}$ & $\begin{array}{l}3620 \pm \\
125\end{array}$ & $\begin{array}{l}3610(3943) \\
4294\end{array}$ & 0 & $-3,9 \pm 1$ & $\begin{array}{l}\text { M.-K. } \\
(1969)\end{array}$ \\
\hline $\mathrm{f}$ & Trézien & $\begin{array}{l}\text { tourbe } \\
\text { dulçaquicole }\end{array}$ & $\begin{array}{l}\text { Gif } \\
714\end{array}$ & $\begin{array}{l}3660 \pm \\
115\end{array}$ & $\begin{array}{l}3690(4000) \\
4300\end{array}$ & 1,5 & $<-2,2 \pm 1$ & $\begin{array}{l}\text { M.-K. } \\
(1969)\end{array}$ \\
\hline$g$ & Argenton (C) & $\begin{array}{l}\text { tourbe } \\
\text { dulçaquicole }\end{array}$ & $\begin{array}{l}\text { Gif } \\
816\end{array}$ & $\begin{array}{l}3970 \pm \\
135\end{array}$ & $\begin{array}{l}4084(4432) \\
4830\end{array}$ & $-0,5$ & $<-4,4 \pm 1$ & $\begin{array}{l}\text { M.-K. } \\
(1969)\end{array}$ \\
\hline $\mathrm{h}$ & Plouescat (base) & $\begin{array}{l}\text { tourbe } \\
\text { dulçaquicole }\end{array}$ & $\begin{array}{l}\text { Gif } \\
711\end{array}$ & $\begin{array}{l}4120 \pm \\
140\end{array}$ & $\begin{array}{l}4234(4635) \\
4976\end{array}$ & 2 & $<-2,7 \pm 1$ & $\begin{array}{l}\text { M.-K. } \\
(1969)\end{array}$ \\
\hline $\mathrm{i}$ & $\begin{array}{l}\text { Plouguerneau } \\
\text { (base) }\end{array}$ & $\begin{array}{l}\text { tourbe } \\
\text { dulçaquicole }\end{array}$ & $\begin{array}{l}\text { Gif } \\
282\end{array}$ & $\begin{array}{l}4250 \pm \\
250\end{array}$ & $\begin{array}{l}4147(4810) \\
5472\end{array}$ & 4 & $<-0,5 \pm 1$ & $\begin{array}{l}\text { M.-K. } \\
(1969)\end{array}$ \\
\hline $\mathrm{j}$ & Brignogan & $\begin{array}{l}\text { tourbe } \\
\text { dulçaquicole }\end{array}$ & Gif 75 & $\begin{array}{l}4980 \pm \\
120\end{array}$ & $\begin{array}{l}5567(5732) \\
5950\end{array}$ & 3 & $<-1,7 \pm 1$ & $\begin{array}{l}\text { M.-K. } \\
(1969)\end{array}$ \\
\hline
\end{tabular}




\begin{tabular}{|c|c|c|c|c|c|c|c|c|}
\hline k & Ploudalmézeau & tourbe littorale & $\begin{array}{l}\text { Gif } \\
766\end{array}$ & $\begin{array}{l}5770 \pm \\
150\end{array}$ & $\begin{array}{l}6288(6581) \\
6933\end{array}$ & $-4,4$ & $-8,8 \pm 1$ & $\begin{array}{l}\text { M.-K. } \\
(1969)\end{array}$ \\
\hline 1 & $\begin{array}{l}\text { Kerlouan } \quad \text { (Le } \\
\text { lerret) }\end{array}$ & $\begin{array}{ll}\text { archéo. } & \text { (base } \\
\text { d'une } & \text { allée } \\
\text { couverte) } & \end{array}$ & - & $\approx 4300$ & $\approx 4300$ & - & $<-5,7$ & $\begin{array}{l}\text { Giot } \\
(1968)\end{array}$ \\
\hline $\mathrm{m}$ & $\begin{array}{l}\text { Le } \quad \text { Kernic } \\
\text { (Roc'hou Braz) }\end{array}$ & $\begin{array}{ll}\text { archéo. } & \text { (base } \\
\text { d'une } & \text { allée } \\
\text { couverte) } & \end{array}$ & - & $\approx 4300$ & $\approx 4300$ & - & $<-3,2$ & $\begin{array}{l}\text { Giot } \\
(1968)\end{array}$ \\
\hline $\mathrm{n}$ & $\begin{array}{l}\text { Guissény } \quad \text { (Le } \\
\text { Curnic) }\end{array}$ & $\begin{array}{l}\text { archéo. } \\
\text { (briquetage proto- } \\
\text { historique) }\end{array}$ & $\begin{array}{l}\text { Gif } \\
160\end{array}$ & $\begin{array}{l}2750 \pm \\
150\end{array}$ & $\begin{array}{l}2469(2888) \\
3266\end{array}$ & 0 & $<-3,9$ & $\begin{array}{l}\text { Giot } \\
(1968)\end{array}$ \\
\hline o & Plouguerneau & $\begin{array}{l}\text { archéo. (base du } \\
\text { Menhir de Men } \\
\text { Ozac'h, St-Cava) }\end{array}$ & - & $\approx 4700$ & $\approx 4700$ & -2 & $<-6,4$ & $\begin{array}{l}\text { Giot } \\
(1968)\end{array}$ \\
\hline $\mathrm{p}$ & $\begin{array}{l}\text { Guissény } \quad \text { (Le } \\
\text { Curnic) }\end{array}$ & $\begin{array}{l}\text { archéo. (foyer sur } \\
\text { une tourbière) }\end{array}$ & $\begin{array}{l}\text { Gif } \\
355\end{array}$ & $\begin{array}{l}5510 \pm \\
250\end{array}$ & $\begin{array}{l}5734(6300) \\
6860\end{array}$ & $-0,1$ & $<-4$ & $\begin{array}{l}\text { Giot } \\
(1970 a)\end{array}$ \\
\hline q & $\begin{array}{l}\text { Guissény } \quad \text { (Le } \\
\text { Curnic) }\end{array}$ & $\begin{array}{l}\text { archéo. (pièce de } \\
\text { monnaie de } \\
\text { Tretricus dans } \\
\text { vivier) }\end{array}$ & - & 1730 & 1730 & - & $<0$ & $\begin{array}{l}\text { Sanquer } \\
(1968)\end{array}$ \\
\hline
\end{tabular}

\section{Sur l'éventualité d'un épisode régressif à l'Âge du Bronze}

Le caractère oscillatoire de la transgression post-glaciaire avancé par la plupart des auteurs dans les années 1960-1970 (Fairbridge, 1961 ; Ters, 1973) a été progressivement remis en cause au cours des deux dernières décennies, grâce à l'acquisition de données de terrain plus précises permettant un meilleur positionnement des anciens niveaux marins. Dans les régions tectoniquement stables, l'absence d'oscillations importantes du niveau global des mers au cours des 6000 dernières années fait désormais l'objet d'un assez large consensus. En s'appuyant sur plusieurs modèles de déglaciation établis avec précision pour la période Holocène, K. Lambeck (1997) a montré que des oscillations de plusieurs mètres en seulement quelques siècles étaient difficilement envisageables. Plus récemment, R.A. Kendall et al. (2008) se sont intéressés à l'impact sur le niveau marin qu'aurait entraîné la vidange brutale du lac Aggasiz-Ojibway vers 8200 cal.BP, considéré comme un événement majeur de la phase de déglaciation holocène. Pour les auteurs, cet épisode n'aurait produit qu'une élévation globale du niveau des mers d'environ $40 \mathrm{~cm}$. W. R. Gehrels (2009) rappelle alors qu'il est improbable que des changements d'ampleur équivalente aient pu se reproduire plus tard au cours de l'Holocène. Selon l'auteur, les courbes dressées en Europe de l'ouest montrent que la variabilité centennale du niveau marin durant la fin de l'Holocène n'a pas dépassé $20 \mathrm{~cm}$ (Gehrels, 2009).

Face à de tels arguments, il convient de s'interroger sur l'hypothèse d'un épisode régressif relatif datant de l'Âge du Bronze reconnu en rade de Brest et dans le nord Finistère. À ce jour, cette hypothèse repose seulement sur deux dates radiocarbone $\left(\mathrm{n}^{\circ} 1\right.$, fig. $4 ; \mathrm{n}^{\circ} \mathrm{b}$, tableau 2$)$ témoignant d'un haut niveau marin proche de l'actuel vers 
3200 cal.BP. Si l'on fait exception de ces deux dates, on peut aisément envisager un mouvement transgressif sans fluctuation majeure depuis 6500 cal.BP jusqu'à aujourd'hui.

Pourtant, plusieurs études récentes réalisées dans la partie orientale de la Manche et en Mer du Nord viennent appuyer les résultats obtenus à ce jour en rade de Brest. Tout d'abord, la courbe dressée par K.-E. Behre (2007) sur les côtes hollandaise, allemande et danoise de la Mer du Nord fait état de plusieurs épisodes régressifs au cours des 5000 dernières années, dont le plus important (épisode R2) se situe à l'Âge du Bronze, entre 3250 et 3000 cal.BP. L'amplitude de cette régression marine est comprise entre 1,6 et $2 \mathrm{~m}$ et ne peut s'expliquer par le simple fait des rejeux glacio-isostatiques. Cette baisse relative du niveau marin correspond également à l'épisode Fenland $\mathrm{V}$ reconnu dans le sud-est de l'Angleterre (Shennan, 1986) qu'une étude plus récente, menée dans la région de Norfolk à partir d'une fonction de transfert utilisant les foraminifères, est venue confirmer (Horton et Edwards, 2005). L'amplitude du mouvement régressif aurait pu atteindre $3 \mathrm{~m}$ selon les auteurs. Enfin, les travaux de E. Gandouin et al. (2007) réalisés dans le bassin de Saint-Omer dans le nord de la France insistent également sur le caractère oscillatoire de la transgression marine holocène. Les sondages effectués dans la partie amont du bassin montrent un remplissage sableux important attribué au Dunkerque I, traduisant un retour à des conditions marécageuses et fluviatiles qui témoignent d'une régression marine entre 3000 et 2500 cal.BP.

Il ne s'agit pas ici de trancher la question du caractère oscillatoire de la transgression marine holocène. Néanmoins, rappelons qu'en Bretagne occidentale, la petite régression de l'Âge du Bronze a toujours été avancée comme élément explicatif de la mise en place des principaux massifs dunaires de la région, à travers l'exondation de vastes surfaces sableuses propices à la déflation éolienne (Hallégouët, 1978; Guilcher et Hallégouët, 1991). Les données archéologiques (tels que les vieux sols qui donnent les éléments post quem pour la mise en place des dunes) montrent que très généralement les systèmes dunaires de Bretagne sont postérieurs au Néolithique et à l'Âge du Bronze, que la plupart des dunes anciennes sont contemporaines de l'Âge du Fer dont des cimetières, des habitats ou des installations industrielles s'y trouvent interstratifiés (Giot, 1968, 1970b; Le Roux, 1971). Récemment, les fouilles archéologiques du site néolithique de Beg ar Louet sur l'île de Molène ont également révélé une phase d'ensablement importante au cours du Bronze moyen (Lyon-3530 [GrA] : 1494-1317 cal.BC), après abandon définitif du site (Pailler et al., 2010). Cette période correspond donc à une phase de changements morphologiques et paysagers importants sur les côtes bretonnes. Toutefois, faute de données suffisantes sur les anciennes positions du niveau marin relatif, il reste difficile d'affirmer à ce jour que ces modifications paysagères trouvent leur origine dans une éventuelle régression marine. L'acquisition de données supplémentaires paraît donc nécessaire pour lever les doutes sur cet épisode régressif, dans l'optique d'une meilleure connaissance paléoenvironnementale et géoarchéologique de la région.

58 Enfin, L. Lespez et al. (2010) ont récemment montré qu'à elles seules, les variations du bilan sédimentaire côtier suffisaient à expliquer les changements géographiques majeurs enregistrés dans la basse vallée de la Dives (Normandie) entre 3000 et 2500 cal.BP. Cette période correspond à une phase d'inondation des marécages par la mer. Cette influence marine n'est pas associée à une pulsation transgressive du niveau marin relatif, mais ferait suite à l'ouverture épisodique de brèches au sein du cordon littoral 
protégeant ces marais, voire à sa destruction pure et simple à la suite d'épisodes météomarins paroxysmaux. Un renforcement des forçages hydrodynamiques à l'Âge du Fer sur le littoral de la Manche est, en effet, avéré puisque des indices de tempêtes extrêmement violentes ont été reconnus en Baie de Seine vers 2700 cal.BP (Sorrel et al., 2009) et en Baie du Mont-Saint-Michel vers 3000 cal. BP (Billeaud et al., 2007). Ces épisodes érosifs s'inscriraient dans une phase de péjoration climatique enregistrée à l'échelle du nord-ouest de l'Europe durant cette période (Van Geel et al., 1996). Ainsi, la plus grande ouverture des marais de la Dives aux influences tidales aurait entraîné la transformation des tourbières dulçaquicoles en véritable schorre. Cet exemple nous incite, une fois de plus, à rester prudent quant aux résultats de l'étude que nous avons mené sur les variations relatives du niveau marin en rade de Brest. Les marais maritimes de la rade sont de dimension modeste et sont protégés par des flèches de galets dont une étude récente a révélé leur relative mobilité au cours des dernières décennies en lien avec l'activité morphogène des tempêtes (Stéphan, 2008). Certes, la rade de Brest présente un caractère d'abri particulièrement prononcé. Néanmoins, on ne peut exclure l'intervention d'épisodes tempétueux ayant modifié temporairement le degré de confinement des zones d'arrière-cordon ainsi que les conditions de sédimentation au sein des marais maritimes, perturbant ainsi les reconstitutions des variations relatives du niveau marin.

\section{Conclusion}

Depuis les travaux de M.-Th. Morzadec-Kerfourn $(1969,1974)$, aucune étude sur les variations holocènes du niveau marin n'avait été réalisée en Bretagne occidentale. L'analyse litho- et biostratigraphique des séquences sédimentaires préservées au sein des trois marais d'arrière-cordon étudiés en rade de Brest a donc permis de réactualiser les données sur l'eustatisme dans ce secteur. La méthode utilisée pour déterminer les anciennes positions du niveau marin s'est appuyée sur la mise au point et l'utilisation d'une fonction de transfert basée sur les assemblages de foraminifères. Cette méthode a permis d'effectuer des estimations du niveau marin avec une précision moyenne d'environ $\pm 50 \mathrm{~cm}$ pour une quinzaine d'échantillons de tourbe et de vase marine datés au ${ }^{14} \mathrm{C}$. Les résultats obtenus présentent une part d'incertitude non négligeable en raison notamment des effets de tassement du matériel sédimentaire qui n'ont pas été corrigés. En outre, les changements plus ou moins importants des conditions de sédimentation au sein des marais maritimes se sont soldés par des fluctuations mineures dans les reconstitutions du niveau marin, perturbant le véritable signal eustatique. Néanmoins, cette méthodologie a permis d'effectuer des estimations du niveau marin à partir de dépôts de vase assez peu compressible, offrant ainsi une meilleure reconstitution du mouvement eustatique fini-holocène par rapport aux études antérieures uniquement basées sur l'analyse des niveaux tourbeux. Malgré quelques écarts significatifs, la courbe obtenue en rade de Brest présente une allure similaire à celle dressée par M.-Th. Morzadec-Kerfourn (1974) dans le nord Finistère. Un épisode régressif est notamment suspecté entre 3200 et 3000 cal.BP. Or, cette baisse particulièrement rapide du niveau marin pose question car elle n'est pas enregistrée de manière systématique sur les autres façades maritimes françaises (Lambeck, 1997 ; Vella et Provansal, 2000), ni même sur les côtes de Belgique (Denys et Beateman, 1995) ou du sud-ouest de l'Angleterre (Shennan et Horton, 2002). Quelques études récentes menées en Mer du Nord (Behre, 2007), dans le sud-est de l'Angleterre 
(Horton et Edwards, 2005) et dans le nord de la France (Gandouin et al., 2007) font toutefois mention d'une régression d'amplitude significative à la fin de l'Âge du Bronze. Notre étude réactualise ainsi le débat autour du caractère oscillatoire de la transgression holocène, sans apporter de réponse définitive. Toutefois, un travail important a été engagé à l'automne 2008 en réalisant de nouveaux carottages dans cinq marais maritimes $\mathrm{du}$ nord Finistère. L'acquisition d'une trentaine de dates radiocarbone supplémentaires devrait nous permettre de proposer une interprétation plus détaillée des variations relatives du niveau marin dans cette région au cours des derniers millénaires.

\section{BIBLIOGRAPHIE}

ALOISI J., MONACO A., PLANCHAIS N., THOMMERET J., THOMMERET Y., 1978. The holocene transgression in the Golfe du Lion, southwestern France : paleogeographic and paléobotanical evolution, Géographie Physique Quaternaire, vol. 32, p. 145-163.

ARMYNOT DU CHÂTELET E., DEBENAY J-P., DEGRÉ D., SAURIAU P-G. 2005. Utilisation des foraminifères benthiques comme indicateurs de paléo-niveaux marins ? Etude du cas de l'anse de l'Aiguillon, C.R. Palévol., n 4, p. 209-223.

BEHRE K. E. 2007. A new Holocene sea-level curve for the southern North Sea, Boreas, vol. 36, p. 82-102.

BILLEAUD I., TESSIER B., LESUEUR P., CALINE B., 2007. Preservation potential of highstand coastal sedimentary bodies in a macrotidal basin: Example from the Bay of Mont-Saint-Michel, NW France, Sedimentary Geology, vol. 202, p. 754-775.

BOOMER I., HORTON B. P. 2006. Holocene relative sea-level movements along the North Norfolk Coast, UK, Palaeogeography, Palaeoclimatology, Palaeoecology, vol. 230, p. 32-51.

CAHOON D. R., REED D. J., DAY J. W., 1995. Estimating shallow subsidence in microtidal salt marshes of the southeastern United States: Kaye and Barghoorn revisited, Marine Geology, vol. 128, p. 1-9.

CLAVÉ B., MASSÉ L., CARBONEL P., TASTET J-P., 2001. Holocene coastal changes and infilling of the La Perroche marsh (French Atlantic coast), Oceanologica Acta, vol. 24, n 4, p. 377-389.

DELANOË Y., PINOT J.-P., 1977. Littoraux et vallées holocènes submergés en baie de Concarneau (Bretagne méridionale), Bulletin de l'Association française pour l'Étude du Quaternaire, vol. 3, p. 27-38. DELIBRIAS G., GIOT P.-R., GOULETQUER P.-L., MORZADEC-KERFOURN M-TH., 1971. Évolution de la ligne de rivage le long du littoral armoricain depuis le Néolithique, Quaternaria, vol. 14, p. 175-179.

DELIBRIAS G., GIOT P.-R., 1970. Inadéquation, hétérogénéité et contamination des échantillons soumis pour les datations radiocarbone, Bulletin de la Société Préhistorique de France, vol. 67, n 5, p. 135-137.

DELIBRIAS G., GUILLIER M.T., 1971. The sea level on the Atlantic coast and the channel for the last 10,000 years by the ${ }^{14} \mathrm{C}$ method, Quaternaria, vol. 14, p. 131-135. 
DENYS L., BAETEMAN C., 1995. Holocene evolution of relative sea level and local mean high water spring tides in Belgium - a first assessment, Marine Geology, vol. 124, p. 1-19.

DUBAR M., ANTHONY E. J., 1995. Holocene environmental change and river-mouth sedimentation in the Baie des Anges, French Riviera, Quaternary Research, vol. 43, n 3, p. 329-343.

DUCHEMIN G., JORISSEN F. J., REDOIS F., DEBENAY J.-P., 2005. Foraminiferal microhabitats in a high marsh: consequences for reconstructing past sea levels, Palaeogeography, Palaeoclimatology, Palaeoecology, vol. 226, p. 167-185.

EDWARDS R. J., 2006. Mid- to late-Holocene relative sea-level change in southwest Britain and the influence of sediment compaction, The Holocene, vol. 16, $\mathrm{n}^{\circ}$ 4, p. 575-587.

EDWARDS R. J., HORTON B. P., 2000. Reconstructing relative sea-level change using UK salt-marsh foraminifera, Marine Geology, vol. 169, p. 41-56.

,- 2006. Developing detailed records of relative sea-level change using a foraminiferal transfer function: an example from North Norfolk, UK, Philosophical Transactions of The Royal Society, vol. 364, p. 973-991.

EDWARDS R. J., VAN DE PLASSCHE O., GEHRELS W. R., WRIGHT A. J., 2004. Assessing sea-level data from Connecticut, USA, using a foraminiferal transfer function for tide level, Marine Micropaleontology, vol. 51, p. 239-255.

EVIN J., 1986. Altérations et datations des sédiments quaternaires, Bulletin de l'Association française pour l'Étude du Quaternaire, vol. 3, p. 205-214.

FAIRBRIDGE R. W., 1961. Eustatic changes in sea level, dans AHRENS L. H., F., RANKAMA K., RUNCORN S. K., Physics and chemistry of the earth, New York, Pergamon Press, vol. 4, p. 99-185.

FATELA F., TABORDA R., 2002. Confidence limits of species proportions in microfossil assemblages, Marine Micropaleontology, vol. 45, p. 169-174.

GANDOUIN E., VAN VLIET-LANOÉ B., FRANQUET E., ANDRIEU-PONEL V., KEEN D. H., PONEL P., MEURISSE M., BRULHET J., BROCANDEL M., 2007. Analyse en haute résolution de l'enregistrement de la transgression holocène dans un secteur subsident du littoral français : le bassin-marais de Saint-Omer (Pas-de-Calais, France), Géologie de la France, vol. 1, p. 11-32.

GEHRELS W. R., 1999. Middle and late Holocene sea_level changes in eastern Maine reconstructed from foraminiferal saltmarsh stratigraphy and AMS ${ }^{14} \mathrm{C}$ dates on basal peat, Quaternary Research, vol. 52, p. 350-359.

,- 2009 . Sea-level changes since the Last Glacial Maximum: an appraisal of the IPCC Fourth Assessment Report, Journal of Quaternary Science, DOI: 10.1002/jqs.1273.

GEHRELS W. R., ROE H. M., CHARMAN D. J., 2001. Foraminifera, testate amoebae and diatoms as sea-level indicators in UK saltmarshes : a quantitative multiproxy approach, Journal of Quaternary Science, vol. 16, $\mathrm{n}^{\circ}$ 3, p. 201-220.

GIOT P.-R., 1968. La Bretagne au péril des mers holocènes, dans La Préhistoire, problèmes et tendances, Paris, CNRS, p. 203-208.

-, 1970a. Chronique des datations radiocarbone armoricaines, Annales de Bretagne, vol. 75,

p. 153-175.

-, 1970b. De l'antiquité des talus et des dunes armoricaines, Penn Ar Bed, vol. 7, nº 60, p. 249-256. 
-, 1992. Le massacre des monuments mégalithiques du Pays Bigouden, Actes du colloque « Le Pays Bigouden, à la croisée des chemins ", Pont-L'Abbé, Edition UBO-CRBC, p. 45-49.

GOULETQUER P.-L., KAYSER O., 1992. Le Mésolithique dans le Pays Bigouden, Actes du colloque « Le Pays Bigouden, à la croisée des chemins ", Pont-L'Abbé, Edition UBO-CRBC, p. 39-44.

GRELLET B., COMBES PH., GRANIER Th., PHILIP H., 1993. Sismotectonique de la France métropolitaine dans son cadre géologique et géophysique, Mémoire NSSGF, 164 p.

GUILBAULT J., CLAGUE J. J., LAPOINTE M., 1996. Foraminiferal evidence for the amount of coeismic subsidence during a late Holocene earthquake on Vancouver Island, west coast of Canada, Quaternary Science Reviews, vol. 15, p. 913-937.

GUILCHER A., 1992. Problems of pleistocene and holocene sea level shifts in western Brittany, dans BILLWITZ K., JÄGER K. D., HERAUSGEBER W. J., KLIEWE H., Jungquartäre landschaftstraüme, aktuelle forschungen zwischen atlantik und tienschan, Edition Springler-Verlag, Berlin, p. 151-156.

GUILCHER A., HALLÉGOUËT B., 1991. Coastal dunes in Brittany and their management, Journal of Coastal Research, vol. 7, n², p. 517-533.

GUILCHER A., VALLANTIN P., ANGRAND J.-P., GALLOY P., 1957. Les cordons littoraux de la rade de Brest, Cahiers Océanographiques, vol. 1, p. 21-54.

HALLÉGOUËT B., 1978. L'évolution des massifs dunaires du pays de Léon, Penn Ar Bed, vol. 11, n 95, p. 417-430.

HINSCHBERGER F., 1970. L'Iroise et les abords d'Ouessant et de Sein. Etude de morphologie et de sédimentologie sous-marines, Association des Publications de la Faculté des Lettres et Sciences Humaines, Université de Caen, $302 \mathrm{p}$.

HORTON B. P., EDWARDS R. J., 2005. The application of local and regional transfer functions to the reconstruction of Holocene sea levels, north Norfolk, England, The Holocene, vol. 15, $\mathrm{n}^{\circ} 2$, p. 216-228.

-, 2006. Quantifying holocene sea-level change using intertidal foraminifera: lessons from the British Isles, Cushman Foundation for foraminiferal Research, Special Publication, nº 40, p. 1-97. HORTON B. P., EDWARDS R. J., LLOYD J. M., 2000. Implications of a microfossil-based transfer function in Holocene sea-level studies, dans SHENNAN, I., ANDREWS, J., Holocene land-ocean interaction and environmental change around the North Sea, Edition Geological Society, Special publication $\mathrm{n}^{\circ} 166$, London, p. 41-54.

JONES R. L., KEEN D. H., BIRNIE J. F., HOLYOAK D. T., 1987. Holocene sea-level changes on Jersey, Progress in Oceanography, vol. 18, p. 177-204.

JUGGINGS G., 2003. C2 data analysis program, version 1.3, Department of Geography, University of Newcastle.

KENDALL R.A., MITROVICA J. X., MILNE G. A., TÖRNQVIST T. E., LI Y., 2008. The sea-level fingerprint of the 8.2 ka climate event, Geology, vol. 36, n 5, p. 423-426.

LABOREL J., MORHANGE C., LAFONT R., LE CAMPION J., LABOREL-DEGUEN F., SARTORETTO S., 1994. Biological evidence of sea-level rise during the last 4500 years on the rocky coasts of continental southwestern France and Corsica, Marine Geology, vol. 120, p. 203-223.

LAMBECK K., 1997. Sea-level change along the French Atlantic and Channel coast since the time of the Last Glacial Maximum, Palaeogeography, Palaeoclimatology, Palaeoecology, vol. 129, p. 1-22. 
LAMBECK K., BARD E., 2000. Sea-level change along the French Mediterranean coast for the past 30000 years, Earth and Planetary Science Letters, vol. 175, p. 203-222.

LE ROUX C.-T., 1971. Une nouvelle perle segmentée en faïence découverte en Bretagne, Bulletin de la Société Préhistorique Française, vol. 68, p. 26-31.

LESPEZ L., CLET-PELLERIN M., DAVIDSON R., HERMIER G., CARPENTIER V., CADOR J.-M., 2010. Middle to Late Holocene landscape changes and geoarchaeological implications in the marshes of the Dives estuary (NW France), Quaternary International, vol. 216, n 1-2, p. 23-40.

LESPEZ L., DALONGEVILLE R., PASTRE J.-F., DARMON F., MATHIEU R., POURSOULIS G., 2002. Late-middle-holocene palaeo-environmental evolution and coastline changes of Malia (Crête), dans FouACHE E. (dir.), Environmental dynamics and history in mediterranean areas, Edition Elsevier, Paris, p. 439-452.

L'HOMER A., BAZILE F., THOMMERET J., THOMMERET Y., 1981. Principales étapes de l'édification du delta du Rhône de 7000 BP à nos jours, variations du niveau marin, Océanis, vol. 7, nº 4, p. 389-408.

LOEBLICH R., TAPPAN H., 1988. Foraminiferal genera and their classification, Department of earth and space science and Center for the study of evolution and the origin of life, University of California, Los Angeles, $970 \mathrm{p}$.

LONG A. J., HUGHES P. D. M., 1995. Mid- and late-Holocene evolution of the Dungeness foreland, UK, Marine Geology, vol. 124, p. 253-271.

LONG A. J., SCAIFE R. G., EDWARDS R. J., 1999. Pine pollen in intertidal sediments from Poole Harbours, UK ; implications for late-Holocene sediment accretion rates and sea-level rise, Quaternary International, vol. 55, p. 3-16.

LONG A. J., WALLER M. P., STUPPLES P., 2006. Driving mechanisms of coastal change: peat compaction and the destruction of ate Holocene coastal wetlands, Marine Geology, vol. 225, p. 63-84.

MARIETTE M., 1971. L'archéologie des dépôts flandriens du Boulonnais, Quaternaria, vol. 14, p. $137-150$.

MASSEY A. C., GEHRELS W. R., CHARMAN D. J., WHITE S. V., 2006. An intertidal foraminifera-based transfer function for reconstructing holocene sea-level change in Southwest England, Journal of Foraminiferal Research, vol. 36, n 3, p. 215-232.

MASSEY A. C., GEHRELS W. R., CHARMAN D. J., MILNE G. A., PELTIER W. R., LAMBECK K., SELBY K. A., 2008. Relative sea-level change and postglacial isostatic adjustment along the coast of south Devon, United Kingdom, Journal of Quaternary Science, vol. 23, n 5, p. 415-433.

MORHANGE C., LABOREL J., HESNARD A., 2001. Changes of relative sea level during the past 5000 years in the ancient harbor of Marseilles, Southern France, Palaeogeography, Palaeoclimatology,

Palaeoecology, vol. 166, p. 319-329.

MORHANGE C., LABOREL J., HESNARD A., PRONE A., 1996. Variation of relative mean sea level during the last 4000 years on the Northern shores of Lacydon, the ancient harbour of Marseilles (Chantier J. Verne), Journal of Coastal Research, vol. 12, n 4, p. 841-849.

MORZADEC-KERFOURN M.-Th., 1969. Variations de la ligne de rivage au cours du post-glaciaire le long de la côte nord du Finistère. Analyses polliniques de tourbes et de dépôts organiques littoraux, Bulletin de l'Association française pour l'Étude du Quaternaire, vol. 4, p. 285-318.

MORZADEC-KERFOURN M.-Th., 1974. Variations de la ligne de rivage armoricaine au Quaternaire. Analyses polliniques de dépôts organiques littoraux, Bulletin de la Société Géologique et Minéralogique de Bretagne, vol. 17, 208 p. 
-, 1975. Évolution paléogéographique du marais de Dol-de-Bretagne (Ille-et-Vilaine) durant le Flandrien, Bulletin de la Société Géologique et Minéralogique de Bretagne, vol. 7, n 1, p. 49-51.

-, 1985. Variations du niveau marin à l'Holocène en Bretagne (France), Eiszeitalter Gegenwart Hannover, vol. 35, p. 15-22.

-, 1995. Coastline changes in the Armorican Massif (France) during the Holocene, Journal of Coastal Research, Special Issue 17, p. 197-203.

,- 2005 . Interaction between sea-level changes and the development of littoral herbaceous vegetation and autotrophic dinoflagellates, Quaternary International, vol. 133/134, p. 137-140. MURRAY J. W., 1971. An atlas of British recent foraminiferids, Edition Heinemann educational books, London, $244 \mathrm{p}$.

-, 1991. Ecology and palaeoecology of benthic foraminifera, Harlow, Longman Group UK Limited, Edition John Wiley \& Sons, 397 p.

PAILLER Y., GANDOIS H., IHUEL E., NICOLAS C., SPARFEL Y., 2010. Le bâtiment en pierres sèches de Beg ar Loued, île Molène (Finistère) : évolution d'une construction du Campaniforme au Bronze ancien, dans Les premiers néolithiques de l'Ouest, Actes du colloque interrégional sur le Néolithique (Le Havre, 2007), supplément Revue Archéologique de l'Ouest, PUR, p. 425-449.

PATTERSON R. T., GUILBAULT J.-P., CLAGUE J. J., 1999. Taphonomy of tidal marsh foraminifera: implications of surface sample thickness for high-resolution sea-level studies, Palaeogeography, Palaeoclimatology, Palaeoecology, vol. 149, p. 199-211.

PINOT J.-P., 1968. Littoraux würmiens submergés à l'ouest de Belle-Île, Bulletin de l'Association française pour l'Étude du Quaternaire, vol. 16, n 3, p. 197-216.

,- 1973. Le quaternaire de la plateforme continentale sud-Armoricaine, Annales scientifiques de l'université de Besançon, vol. 3, n²1, p. 87-92.

PIRAZZOLI P. A., 1976. Les variations du niveau marin depuis 2000 ans, Mémoires du laboratoire de géomorphologie de l'école pratique des hautes études, Dinard, $421 \mathrm{p}$.

-, 1996. Sea-level Changes. The Last 20000 years, Edition John Wiley \& Sons, Chichester, 211 p. PONTEE N. I., TASTET J.-P., MASSÉ L., 1998. Morpho-sedimentary evidence of Holocene coastal changes near the mouth of the Gironde and on the Medoc Peninsula, SW France, Oceanologica Acta, vol. 21, $\mathrm{n}^{\circ} 2$, p. 243-261.

REGNAULD H., JENNINGS S. C., DELANEY C., LEMASSON L., 1996. Holocene sea-level variations and geomorphological response: an example from northern Brittany (France), Quaternary Science Reviews, vol. 15, p. 781-787.

REIMER P. J., BAILLIE M. G. L, BARD E., BAYLISS A., BECK J. W., BLACKWELL P. G., BRONK RAMSEY C., BUCK C. E., BURR G. S., EDWARDS R. L., FRIEDRICH M., GROOTES P. M., GUILDERSON T. P., HAJDAS I., HEATON T.J., HOGG A. G., HUGHEN K. A., KAISER K. F., KROMER B., MCCORMAC F. G., MANNING S.W., REIMER R. W., RICHARDS D.A., SOUTHON J.R., TALAMO S., TURNEY C. S. M., VAN DER PLICHT J., WEYHENMEYER C. E., 2009. IntCal09 and Marine09 Radiocarbon Age Calibration Curves, 0-50,000 Years cal.BP, Radiocarbon, vol. 51, p. 1111-1150.

SANQUER R., 1968. Découvertes récentes aux environs de Brest (Période Romaine) : le réservoir à poissons du Curnic en Guisseny, Annales de Bretagne, LXXV, p. 246-265.

SCOTT D. F., MEDIOLI F. S., 1978. Vertical zonations of marsh foraminifera as accurate indicators of former sea-levels, Nature, vol. 272, p. 528-531. 
SCOTT D. F., MEDIOLI F. S., 1980. Living vs. total foraminifera populations: their relative usefulness in paleoecology, Journal of Paleontology, vol. 54, p. 814-831.

SCOTT D. B., COLLINS E. S., DUGGAN J., ASIOLI A., SAITO T., HASEGAWA S., 1996. Pacific Rim marsh foraminiferal distributions: implications for sea-level studies, Journal of Coastal Research, vol. 12, nº 4, p. 850-861.

SHENNAN I., 1986. Flandrian sea-level changes in the Fenland. II: Tendencies of sea-level movement, altitudinal changes, and local and regional factors, Journal of Quaternary Science, vol. 1, p. 155-179.

SHENNAN I., HORTON B. P., 2002. Holocene land- and sea-level changes in Great Britain, Journal of Quaternary Science, vol. 17, $\mathrm{n}^{\circ}$ 5-6, p. 511-526.

SORREL P., TESSIER B., DEMORY F., DELSINNE N., MOUAZE D., 2009. Evidence for millennial-scale climatic events in the sedimentary infilling of a macrotidal estuarine system, the Seine estuary (NW France), Quaternary Science Reviews, vol. 28, p. 499-516.

SOUTHALL K. E., GEHRELS R. W., HAYWARD B. W., 2006. Foraminifera in New Zealand salt marsh and their suitability as sea-level indicators, Marine Micropaleontology, vol. 60, p. 167-179.

STÉPHAN P., 2008. Les flèches de galets de Bretagne : morphodynamiques passée, présente et prévisible, thèse de doctorat en géographie physique, Université de Bretagne Occidentale, Brest, 548 p.

STUIVER M., REIMER P. J., 1993. Extended ${ }^{14} \mathrm{C}$ data base and revised CALIB.3.0. ${ }^{14} \mathrm{C}$ age calibration program, Radiocarbon, vol. 35, p. 215-230.

TERS M., 1973. Les variations du niveau marin depuis 10000 ans le long du littoral atlantique français, dans Edition CNRS, Le Quaternaire, Géodynamique, Stratigraphie et Environnement, Paris, p. 114-135.

,- 1986 . Variations in Holocene sea level on the french Atlantic coast and their climatic significance, dans RAMPINO M.R., SANDERS J. E., NEWMAN W. S., KÖNIGSSON L. K., Climate: history, periodicity and predictability, Edition Van Nostrand Reinhold, New York, p. 204-237.

VAN DE PLASSCHE O., 1991. Coastal submergence of the Netherlands, NW Brittany (France), Delmarva Peninsula (VA, USA) and Connecticut (USA) during the last 5500 to 7500 sidereal years, dans SABADINI R. (dir.), Glacial isostasy, sea level and mantle rheology, Edition Kluwer, Dordrecht, p. $285-300$.

VAN GEEL B., BUURMAN J., WATERBOLK H. T., 1996. Archeological and palaeoecological indications of an abrupt climate change in the Netherlands, and evidence for climatological teleconnections around 2650 BP, Journal of Quaternary Science, vol. 11, n 6, p. 451-460.

VAN VLIET-LANOË B., BONNET S., HALLÉGOUËT B., LAURENT M., 1997. Neotectonic and seismic activity in the armorican and cornubian massifs : regional stress field with glacio-isostatic influence ?, Journal of Geodynamics, vol. 24, n 1-4, p. 219-139.

VELLA C., 1999. Perception et évaluation de la mobilité du littoral holocène sur la marge orientale du delta du Rhône, Thèse de doctorat de Géographie, Université de Provence Aix-Marseille, 225 p.

VELLA C., 2004. Le rôle de la mer : position du niveau marin et du trait de côte depuis 6000 ans, Bulletin archéologique de Provence, Suppl. 2, p. 79-90.

VELLA C., FLEURY T.-J., RACCASI G., PROVANSAL M., SABATIER F., BOURCIER M., 2005. Evolution of the Rhône delta plain in the Holocene, Marine Geology, vol. 222/223, p. 235-265.

VELLA C., PROVANSAL M., 2000. Relative sea-level rise and neotectonic events during the last $6500 \mathrm{yr}$ on the southern eastern Rhône delta, France, Marine Geology, vol. 170, p. 27-39. 
VISSET L., BERNARD J., 2006. Evolution du littoral et du paysage, de la presqu'île de Rhuys à la rivière d'Etel (Massif armoricain - France), du Néolithique au Moyen Âge, Revue d'archéométrie, vol. 30, p. 143-156.

WALLER M. P., LONG A. J., 2003. Holocene coastal evolution and sea-level change on the southern coast of England: a review, Journal of Quaternary Science, vol. 18, n³/4, p. 351-359.

WALLER M. P., LONG A. J., LONG D., INNES J. B., 1999. Patterns and processes in the development of coastal mire vegetation: multi-site investigations from Walland Marsh, Southeast England, Quaternary Science Reviews, vol. 18, p. 1419-1444.

WOODROFFE S. A., 2009. Recognising subtidal foraminiferal assemblages: implications for quantitative sea-level reconstructions using a foraminifera-based transfert function, Journal of Quaternary Science, vol. 24, n 3, p. 215-223.

ZONG Y., HORTON B. P., 1999. Diatom-based tidal-level transfer functions as an aid in reconstructing Quaternary history of sea-level movements in the UK, Journal of Quaternary Science, vol. 14, $\mathrm{n}^{\circ} 2$, p. 153-167.

\section{RÉSUMÉS}

Afin d'apporter des données nouvelles sur les variations relatives du niveau marin en Bretagne occidentale durant l'Holocène, une analyse biostratigraphique et lithostratigraphique a été entreprise dans trois marais maritimes d'arrière-cordon situés en rade de Brest. La mise au point d'une fonction de transfert basée sur les foraminifères a permis de positionner les anciens niveaux marins avec une précision moyenne de $\pm 51 \mathrm{~cm}$ pour une quinzaine d'échantillons de vase marine et de tourbe littorale datés au ${ }^{14} \mathrm{C}$ et prélevés le long de quatre sondages de référence. Les résultats obtenus permettent de distinguer quatre grandes étapes dans le mouvement transgressif enregistré au cours des 6000 dernières années en rade de Brest. Entre 6250 et 5500 cal.BP, le niveau marin relatif montre une relative stabilité, se situant à $5 \mathrm{~m}$ environ sous le niveau actuel. Entre 5500 et 3200 cal.BP, la transgression marine reprend à une vitesse moyenne de $2 \mathrm{~mm} / \mathrm{an}$ et amène le niveau marin relatif à une position légèrement inférieure à l'actuel. Entre 3200 et 2800 cal.BP, une petite régression marine relative est suspectée, conduisant à une baisse du niveau de la mer d'environ $2 \mathrm{~m}$. Après 2800 cal.BP, le mouvement transgressif reprend à un rythme décroissant jusqu'à nos jours. Ces résultats sont en accord avec les données acquises par M.-Th. Morzadec-Kerfourn $(1969,1974)$ dans le nord Finistère. Néanmoins, l'épisode régressif situé aux environs de 3000 cal.BP pose question. Si quelques études récentes réalisées en Mer du Nord et dans la partie orientale de la Manche en font état, cette régression n'est pas enregistrée de manière systématique sur les différentes façades maritimes françaises, ni même sur les côtes de Belgique ou du sud-ouest de l'Angleterre.

The lithostratigraphy and biostratigraphy of a three back-barrier sediment sequences in Bay of Brest are examined to reconstruct the holocene sea-level history in the western part of Brittany. A salt-marsh foraminifera-based transfer function is used to assess palaeo-sea-level positions with a precision of $\pm 0,51 \mathrm{~m}$. The transfer function is applied to foraminiferal assemblages from four cores, providing 16 news sea-level index points from western Brittany. Our data suggest a relative stability of relative sea-level between 6250 and $5500 \mathrm{cal}$. yr BP, followed by a period of sea-level rise (about $2 \mathrm{~mm} / \mathrm{yr}$ ) between 5500 and 3200 cal.yr BP. A decrease of relative sea-level with a magnitude of $2 \mathrm{~m}$ is suspected between 3200 and 2800 cal.yr BP. After 2800 cal.yr BP, a new period of sea-level rise is recorded, slowing until today. These results are in agreement with 
the curve of Morzadec-Kerfourn $(1969,1974)$ from the northwestern part of Brittany. However, the period of relative sea-level fall around 3000 cal.yr BP is questioned. Although this regression phase is recorded in the North Sea and in the oriental part of the English Channel, this event is recognized neither along the other French coasts, nor along the south western coast of England.

INDEX

Index géographique : Brest (rade de), Bretagne, Finistère

Keywords : Holocene, Quaternary, sea-level rise, foraminifera

Mots-clés : Holocène, niveau marin, Quaternaire, foraminifères

\section{AUTEUR}

\section{PIERRE STÉPHAN}

Géomer - UMR 6554 CNRS LETG (Université de Bretagne Occidentale), Institut Universitaire Européen de la Mer - Technopole Brest-Iroise, place Nicolas-Copernic - 29280 Plouzané, France pierre.stephan@univ-brest.fr 